\title{
Understanding Galaxy Formation and Evolution
}

\author{
Vladimir Avila-Reese ${ }^{1}$ \\ Instituto de Astronomía, Universidad Nacional Autónoma de México, A.P. 70-264, \\ 04510, México,D.F. avila@astroscu.unam.mx
}

The old dream of integrating into one the study of micro and macrocosmos is now a reality. Cosmology, astrophysics, and particle physics intersect in a scenario (but still not a theory) of cosmic structure formation and evolution called $\Lambda$ Cold Dark Matter $(\Lambda \mathrm{CDM})$ model. This scenario emerged mainly to explain the origin of galaxies. In these lecture notes, I first present a review of the main galaxy properties, highlighting the questions that any theory of galaxy formation should explain. Then, the cosmological framework and the main aspects of primordial perturbation generation and evolution are pedagogically detached. Next, I focus on the "dark side" of galaxy formation, presenting a review on $\Lambda \mathrm{CDM}$ halo assembling and properties, and on the main candidates for non-baryonic dark matter. It is shown how the nature of elemental particles can influence on the features of galaxies and their systems. Finally, the complex processes of baryon dissipation inside the non-linearly evolving CDM halos, formation of disks and spheroids, and transformation of gas into stars are briefly described, remarking on the possibility of a few driving factors and parameters able to explain the main body of galaxy properties. A summary and a discussion of some of the issues and open problems of the $\Lambda \mathrm{CDM}$ paradigm are given in the final part of these notes.

\section{Introduction}

Our vision of the cosmic world and in particular of the whole Universe has been changing dramatically in the last century. As we will see, galaxies were repeatedly the main protagonist in the scene of these changes. It is about 80 years since E. Hubble established the nature of galaxies as gigantic selfbound stellar systems and used their kinematics to show that the Universe as a whole is expanding uniformly at the present time. Galaxies, as the building blocks of the Universe, are also tracers of its large-scale structure and of its evolution in the last 13 Gyrs or more. By looking inside galaxies we find that they are the arena where stars form, evolve and collapse in constant 
interaction with the interstellar medium (ISM), a complex mix of gas and plasma, dust, radiation, cosmic rays, and magnetics fields. The center of a significant fraction of galaxies harbor supermassive black holes. When these "monsters" are fed with infalling material, the accretion disks around them release, mainly through powerful plasma jets, the largest amounts of energy known in astronomical objects. This phenomenon of Active Galactic Nuclei (AGN) was much more frequent in the past than in the present, being the high-redshift quasars (QSO's) the most powerful incarnation of the AGN phenomenon. But the most astonishing surprise of galaxies comes from the fact that luminous matter (stars, gas, AGN's, etc.) is only a tiny fraction $(\sim 1-5 \%)$ of all the mass measured in galaxies and the giant halos around them. What this dark component of galaxies is made of? This is one of the most acute enigmas of modern science.

Thus, exploring and understanding galaxies is of paramount interest to cosmology, high-energy and particle physics, gravitation theories, and, of course, astronomy and astrophysics. As astronomical objects, among other questions, we would like to know how do they take shape and evolve, what is the origin of their diversity and scaling laws, why they cluster in space as observed, following a sponge-like structure, what is the dark component that predominates in their masses. By answering to these questions we would able also to use galaxies as a true link between the observed universe and the properties of the early universe, and as physical laboratories for testing fundamental theories.

The content of these notes is as follows. In $\S 2$ a review on main galaxy properties and correlations is given. By following an analogy with biology, the taxonomical, anatomical, ecological and genetical study of galaxies is presented. The observational inference of dark matter existence, and the baryon budget in galaxies and in the Universe is highlighted. Section 3 is dedicated to a pedagogical presentation of the basis of cosmic structure formation theory in the context of the $\Lambda$ Cold Dark Matter $(\Lambda \mathrm{CDM})$ paradigm. The main questions to be answered are: why CDM is invoked to explain the formation of galaxies? How is explained the origin of the seeds of present-day cosmic structures? How these seeds evolve? In $\S 4$ an updated review of the main results on properties and evolution of CDM halos is given, with emphasis on the aspects that influence the propertied of the galaxies expected to be formed inside the halos. A short discussion on dark matter candidates is also presented (§§4.2). The main ingredients of disk and spheroid galaxy formation are reviewed and discussed in $\S 5$. An attempt to highlight the main drivers of the Hubble and color sequences of galaxies is given in $§ \S 5.3$. Finally, some selected issues and open problems in the field are resumed and discussed in $\S 6$.

\section{Galaxy properties and correlations}

During several decades galaxies were considered basically as self-gravitating stellar systems so that the study of their physics was a domain of Galactic 
Dynamics. Galaxies in the local Universe are indeed mainly conglomerates of hundreds of millions to trillions of stars supported against gravity either by rotation or by random motions. In the former case, the system has the shape of a flattened disk, where most of the material is on circular orbits at radii that are the minimal ones allowed by the specific angular momentum of the material. Besides, disks are dynamically fragile systems, unstable to perturbations. Thus, the mass distribution along the disks is the result of the specific angular momentum distribution of the material from which the disks form, and of the posterior dynamical (internal and external) processes. In the latter case, the shape of the galactic system is a concentrated spheroid/ellipsoid, with mostly (disordered) radial orbits. The spheroid is dynamically hot, stable to perturbations. Are the properties of the stellar populations in the disk and spheroid systems different?

\section{Stellar populations}

Already in the 40's, W. Baade discovered that according to the ages, metallicities, kinematics and spatial distribution of the stars in our Galaxy, they separate in two groups: 1) Population I stars, which populate the plane of the disk; their ages do not go beyond $10 \mathrm{Gyr}$-a fraction of them in fact are young $\left(\lesssim 10^{6}\right.$ yr) luminous O,B stars mostly in the spiral arms, and their metallicites are close to the solar one, $Z \approx 2 \% ; 2$ ) Population II stars, which are located in the spheroidal component of the Galaxy (stellar halo and partially in the bulge), where velocity dispersion (random motion) is higher than rotation velocity (ordered motion); they are old stars (>10 Gyr) with very low metallicities, on the average lower by two orders of magnitude than Population I stars. In between Pop's I and II there are several stellar subsystems. ${ }^{1}$.

Stellar populations are true fossils of the galaxy assembling process. The differences between them evidence differences in the formation and evolution of the galaxy components. The Pop II stars, being old, of low metallicity, and dominated by random motions (dynamically hot), had to form early in the assembling history of galaxies and through violent processes. In the meantime, the large range of ages of Pop I stars, but on average younger than the Pop II stars, indicates a slow star formation process that continues even today in the disk plane. Thus, the common wisdom says that spheroids form early in a violent collapse (monolithic or major merger), while disks assemble by continuous infall of gas rich in angular momentum, keeping a self-regulated SF process.

\footnotetext{
${ }^{1}$ Astronomers suspect also the existence of non-observable Population III of pristine stars with zero metallicities, formed in the first molecular clouds $\sim 410^{8}$ yrs $(z \sim 20)$ after the Big Bang. These stars are thought to be very massive, so that in scaletimes of 1 Myr they exploded, injected a big amount of energy to the primordial gas and started to reionize it through expanding cosmological HII regions (see e.g., 20] 27] for recent reviews on the subject).
} 
Galaxies are not only conglomerates of stars. The study of galaxies is incomplete if it does not take into account the ISM, which for late-type galaxies accounts for more mass than that of stars. Besides, it is expected that in the deep past, galaxies were gas-dominated and with the passing of time the cold gas was being transformed into stars. The ISM is a turbulent, nonisothermal, multi-phase flow. Most of the gas mass is contained in neutral instable HI clouds $\left(10^{2}<T<10^{4} \mathrm{~K}\right)$ and in dense, cold molecular clouds $\left(T<10^{2} \mathrm{~K}\right)$, where stars form. Most of the volume of the ISM is occuppied by diffuse $\left(n \approx 0.1 \mathrm{~cm}^{-3}\right)$, warm-hot $\left(T \approx 10^{4}-10^{5} \mathrm{~K}\right)$ turbulent gas that confines clouds by pressure. The complex structure of the ISM is related to (i) its peculiar thermodynamical properties (in particular the heating and cooling processes), (ii) its hydrodynamical and magnetic properties which imply development of turbulence, and (iii) the different energy input sources. The star formation unities (molecular clouds) appear to form during large-scale compression of the diffuse ISM driven by supernovae (SN), magnetorotational instability, or disk gravitational instability (e.g., [7). At the same time, the energy input by stars influences the hydrodynamical conditions of the ISM: the star formation results self-regulated by a delicate energy (turbulent) balance.

Galaxies are true "ecosystems" where stars form, evolve and collapse in constant interaction with the complex ISM. Following a pedagogical analogy with biological sciences, we may say that the study of galaxies proceeded through taxonomical, anatomical, ecological and genetical approaches.

\subsection{Taxonomy}

As it happens in any science, as soon as galaxies were discovered, the next step was to attempt to classify these news objects. This endeavor was taken on by E. Hubble. The showiest characteristics of galaxies are the bright shapes produced by their stars, in particular those most luminous. Hubble noticed that by their external look (morphology), galaxies can be divided into three principal types: Ellipticals (E, from round to flattened elliptical shapes), Spirals ( $\mathrm{S}$, characterized by spiral arms emanating from their central regions where an spheroidal structure called bulge is present), and Irregulars (Irr, clumpy without any defined shape). In fact, the last two classes of galaxies are diskdominated, rotating structures. Spirals are subdivided into $\mathrm{Sa}, \mathrm{Sb}, \mathrm{Sc}$ types according to the size of the bulge in relation to the disk, the openness of the winding of the spiral arms, and the degree of resolution of the arms into stars (in between the arms there are also stars but less luminous than in the arms). Roughly $40 \%$ of S galaxies present an extended rectangular structure (called bar) further from the bulge; these are the barred Spirals (SB), where the bar is evidence of disk gravitational instability.

From the physical point of view, the most remarkable aspect of the morphological Hubble sequence is the ratio of spheroid (bulge) to total luminosity. 
This ratio decreases from 1 for the Es, to $\sim 0.5$ for the so-called lenticulars (S0), to $\sim 0.5-0.1$ for the Ss, to almost 0 for the Irrs. What is the origin of this sequence? Is it given by nature or nurture? Can the morphological types change from one to another and how frequently they do it? It is interesting enough that roughly half of the stars at present are in galaxy spheroids (Es and the bulges of S0s and Ss), while the other half is in disks (e.g., [11]), where some fraction of stars is still forming.

\subsection{Anatomy}

The morphological classification of galaxies is based on their external aspect and it implies somewhat subjective criteria. Besides, the "showy" features that characterize this classification may change with the color band: in blue bands, which trace young luminous stellar populations, the arms, bar and other features may look different to what it is seen in infrared bands, which trace less massive, older stellar populations. We would like to explore deeper the internal physical properties of galaxies and see whether these properties correlate along the Hubble sequence. Fortunately, this seems to be the case in general so that, in spite of the complexity of galaxies, some clear and sequential trends in their properties encourage us to think about regularity and the possibility to find driving parameters and factors beyond this complexity.

Figure 1 below resumes the main trends of the "anatomical" properties of galaxies along the Hubble sequence.

The advent of extremely large galaxy surveys made possible massive and uniform determinations of global galaxy properties. Among others, the Sloan Digital Sky Survey $\left(\mathrm{SDSS}^{2}\right)$ and the Two-degree Field Galaxy Redshift Survey $\left(2 \mathrm{dFGRS}^{3}\right)$ currently provide uniform data already for around $10^{5}$ galaxies in limited volumes. The numbers will continue growing in the coming years. The results from these surveys confirmed the well known trends shown in Fig. 1] moreover, it allowed to determine the distributions of different properties. Most of these properties present a bimodal distribution with two main sequences: the red, passive galaxies and the blue, active galaxies, with a fraction of intermediate types (see for recent results [68, 6, 114, 34, 127, and more references therein). The most distinct segregation in two peaks is for the specific star formation rate $\left(\dot{M}_{s} / M_{s}\right)$; there is a narrow and high peak of passive galaxies, and a broad and low peak of star forming galaxies. The two sequences are also segregated in the luminosity function: the faint end is dominated by the blue, active sequence, while the bright end is dominated by the red, passive sequence. It seems that the transition from one sequence to the other happens at the galaxy stellar mass of $\sim 3 \times 10^{10} \mathrm{M}_{\odot}$.

\footnotetext{
${ }^{2}$ www.sdss.org/sdss.html

3 www.aao.gov.au/2df/
} 


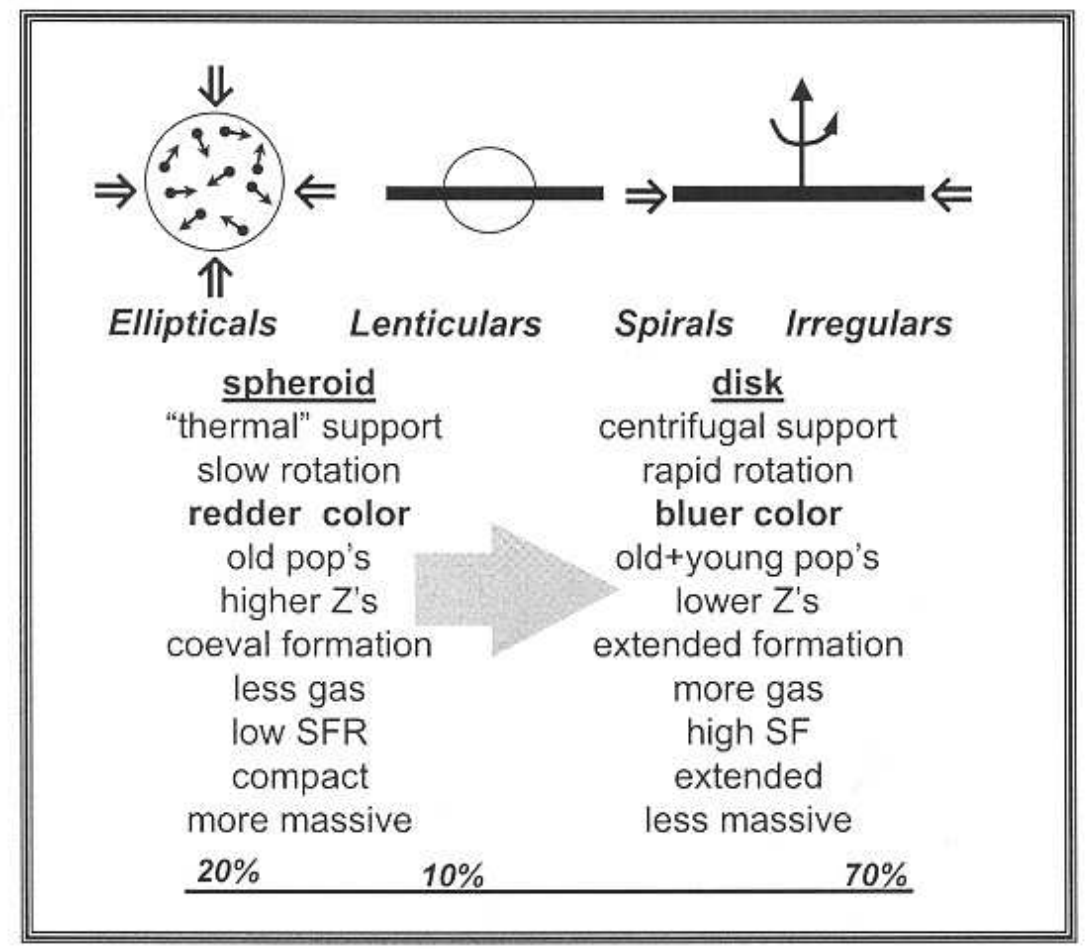

Fig. 1. Main trends of physical properties of galaxies along the Hubble morphological sequence. The latter is basically a sequence of change of the spheroid-to-disk ratio. Spheroids are supported against gravity by velocity dispersion, while disks by rotation.

\section{The hidden component}

Under the assumption of Newtonian gravity, the observed dynamics of galaxies points out to the presence of enormous amounts of mass not seen as stars or gas. Assuming that disks are in centrifugal equilibrium and that the orbits are circular (both are reasonable assumptions for non-central regions), the measured rotation curves are good tracers of the total (dynamical) mass distribution (Fig. 2). The mass distribution associated with the luminous galaxy (stars+gas) can be inferred directly from the surface brightness (density) profiles. For an exponential disk of scalelength $R_{d}(=3 \mathrm{kpc}$ for our Galaxy), the rotation curve beyond the optical radius $\left(R_{o p t} \approx 3.2 R_{d}\right)$ decreases as in the Keplerian case. The observed HI rotation curves at radii around and beyond $R_{\text {opt }}$ are far from the Keplerian fall-off, implying the existence of hidden mass called dark matter (DM) 99, 18. The fraction of DM increases with radius.

It is important to remark the following observational facts: 


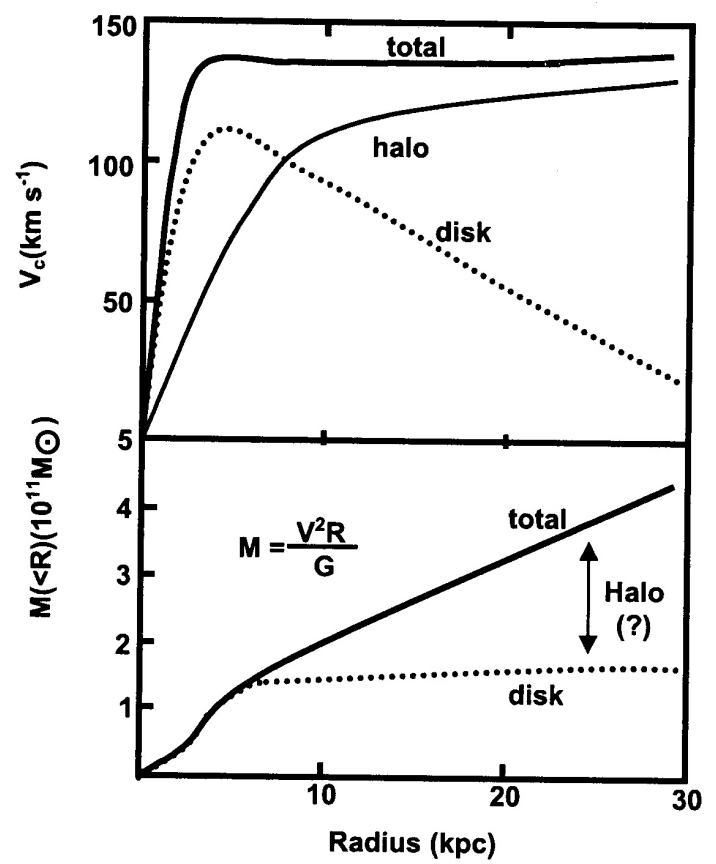

Fig. 2. Under the assumption of circular orbits, the observed rotation curve of disk galaxies traces the dynamical (total) mass distribution. The outer rotation curve of a nearly exponential disk decreases as in the Keplerian case. The observed rotation curves are nearly flat, suggesting the existence of massive dark halos.

- the outer rotation curves are not universally flat as it is assumed in hundreds of papers. Following, Salucci \& Gentile [101, let us define the average value of the rotation curve logarithmic slope, $\nabla \equiv(d \log V / d \log R)$ between two and three $R_{d}$. A flat curve means $\nabla=0$; for an exponential disk without DM, $\nabla=-0.27$ at $3 R_{s}$. Observations show a large range of values for the slope: $-0.2 \leq \nabla \leq 1$

- the rotation curve shape $(\nabla)$ correlates with the luminosity and surface brightness of galaxies 95, 123, 132]: it increases according the galaxy is fainter and of lower surface brightness

- at the optical radius $R_{\text {opt }}$, the DM-to-baryon ratio varies from $\approx 1$ to 7 for luminous high-surface brightness to faint low-surface brightness galaxies, respectively

- the roughly smooth shape of the rotation curves implies a fine coupling between disk and DM halo mass distributions 24

The HI rotation curves extend typically to $2-5 R_{\text {opt }}$. The dynamics at larger radii can be traced with satellite galaxies if the satellite statistics allows for that. More recently, the technique of (statistical) weak lensing around 
galaxies began to emerge as the most direct way to trace the masses of galaxy halos. The results show that a typical $L_{*}$ galaxy (early or late) with a stellar mass of $M_{s} \approx 6 \times 10^{10} \mathrm{M}_{\odot}$ is surrounded by a halo of $\approx 2 \times 10^{12} \mathrm{M}_{\odot}$ ( 80 and more references therein). The extension of the halo is typically $\approx 200-250 \mathrm{kpc}$. These numbers are very close to the determinations for our own Galaxy.

The picture has been confirmed definitively: luminous galaxies are just the top of the iceberg (Fig. 3). The baryonic mass of (normal) galaxies is only $\sim 3-5 \%$ of the DM mass in the halo! This fraction could be even lower for dwarf galaxies (because of feedback) and for very luminous galaxies (because the gas cooling time $>$ Hubble time). On the other hand, the universal baryonto-DM fraction $\left(\Omega_{B} / \Omega_{D M} \approx 0.04 / 0.022\right.$, see below) is $f_{B, U n} \approx 18 \%$. Thus, galaxies are not only dominated by DM, but are much more so than the average in the Universe! This begs the next question: if the majority of baryons is not in galaxies, where it is? Recent observations, based on highly ionized absorption lines towards low redshfit luminous AGNs, seem to have found a fraction of the missing baryons in the interfilamentary warm-hot intergalactic

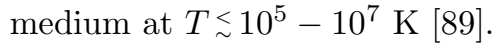

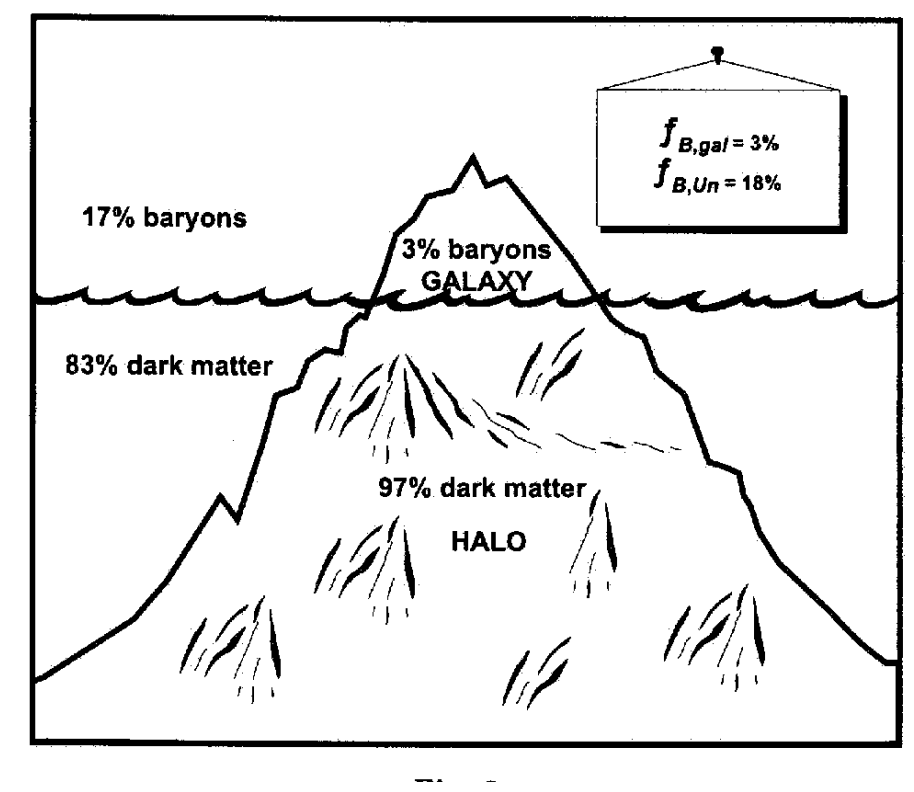

Fig. 3. Galaxies are just the top of the iceberg. They are surrounded by enormous DM halos extending 10-20 times their sizes, where baryon matter is only less than $5 \%$ of the total mass. Moreover, galaxies are much more DM-dominated than the average content of the Universe. The corresponding typical baryon-to-DM mass ratios are given in the inset. 
Global baryon inventory: The different probes of baryon abundance in the Universe (primordial nucleosynthesis of light elements, the ratios of odd and even CMBR acoustic peaks heights, absorption lines in the Ly $\alpha$ forest) have been converging in the last years towards the same value of the baryon density: $\Omega_{b} \approx 0.042 \pm 0.005$. In Table 1 below, the densities $\left(\Omega^{\prime} \mathrm{s}\right)$ of different baryon components at low redshfits and at $z>2$ are given (from [48] and [89]).

Table 1. Abundances of the different baryon components $(h=0.7)$

\begin{tabular}{lr}
\hline Component & Contribution to $\Omega$ \\
\hline Low redshifts & \\
Galaxies: stars & $0.0027 \pm 0.0005$ \\
Galaxies: HI & $(4.2 \pm 0.7) \times 10^{-4}$ \\
Galaxies: $\mathrm{H}_{2}$ & $(1.6 \pm 0.6) \times 10^{-4}$ \\
Galaxies: others & $(\approx 2.0) \times 10^{-4}$ \\
Intracluster gas & $0.0018 \pm 0.0007$ \\
IGM: (cold-warm) & $0.013 \pm 0.0023$ \\
IGM: (warm-hot) & $\approx 0.016$ \\
$z>2$ & $>0.035$ \\
Ly $\alpha$ forest clouds & \\
\hline
\end{tabular}

The present-day abundance of baryons in virialized objects (normal stars, gas, white dwarfs, black holes, etc. in galaxies, and hot gas in clusters) is therefore $\Omega_{B} \approx 0.0037$, which accounts for $\approx 9 \%$ of all the baryons at low redshifts. The gas in not virialized structures in the Intergalactic Medium (cold-warm Ly $\alpha / \beta$ gas clouds and the warm-hot phase) accounts for $\approx 73 \%$ of all baryons. Instead, at $z>2$ more than $88 \%$ of the universal baryonic fraction is in the Ly $\alpha$ forest composed of cold HI clouds. The baryonic budget's outstanding questions: Why only $\approx 9 \%$ of baryons are in virialized structures at the present epoch?

\subsection{Ecology}

The properties of galaxies vary systematically as a function of environment. The environment can be relatively local (measured through the number of nearest neighborhoods) or of large scale (measured through counting in defined volumes around the galaxy). The morphological type of galaxies is earlier in the locally denser regions (morphology-density relation), the fraction of ellipticals being maximal in cluster cores [40] and enhanced in rich 96] and poor groups. The extension of the morphology-density relation to low local-density environment (cluster outskirts, low mass groups, field) has been a matter of debate. From an analysis of SDSS data, [54 have found that (i) in the sparsest regions both relations flatten out, (ii) in the intermediate density regions (e.g., cluster outskirts) the intermediate-type galaxy (mostly S0s) fraction increases 
towards denser regions whereas the late-type galaxy fraction decreases, and (iii) in the densest regions intermediate-type fraction decreases radically and early-type fraction increases. In a similar way, a study based on 2dFGRS data of the luminosity functions in clusters and voids shows that the population of faint late-type galaxies dominates in the latter, while, in contrast, very bright early-late galaxies are relatively overabundant in the former 34. This and other studies suggest that the origin of the morphology-density (or morphology-radius) relation could be a combination of (i) initial (cosmological) conditions and (ii) of external mechanisms (ram-pressure and tidal stripping, thermal evaporation of the disk gas, strangulation, galaxy harassment, truncated star formation, etc.) that operate mostly in dense environments, where precisely the relation steepens significantly.

The morphology-environment relation evolves. It systematically flattens with $z$ in the sense that the grow of the early-type $(\mathrm{E}+\mathrm{S} 0)$ galaxy fraction with density becomes less rapid (97] and more references therein) the main change being in the high-density population fraction. Postman et al. conclude that the observed flattening of the relation up to $z \sim 1$ is due mainly to a deficit of S0 galaxies and an excess of Sp+Irr galaxies relative to the local galaxy population; the $\mathrm{E}$ fraction-density relation does not appear to evolve over the range $0<z<1.3$ ! Observational studies show that other properties besides morphology vary with environment. The galaxy properties most sensitive to environment are the integral color and specific star formation rate (e.g. 68 114,127 . The dependences of both properties on environment extend typically to lower densities than the dependence for morphology. These properties are tightly related to the galaxy star formation history, which in turn depends on internal formation/evolution processes related directly to initial cosmological conditions as well as to external astrophysical mechanisms able to inhibit or induce star formation activity.

\subsection{Genetics}

Galaxies definitively evolve. We can reconstruct the past of a given galaxy by matching the observational properties of its stellar populations and ISM with (parametric) spectro-photo-chemical models (inductive approach). These are well-established models specialized in following the spectral, photometrical and chemical evolution of stellar populations formed with different gas infall rates and star formation laws (e.g. [16 and the references therein). The inductive approach allowed to determine that spiral galaxies as our Galaxy can not be explained with closed-box models (a single burst of star formation); continuous infall of low-metallicity gas is required to reproduce the local and global colors, metal abundances, star formation rates, and gas fractions. On the other hand, the properties of massive ellipticals (specially their high $\alpha$-elements/Fe ratios) are well explained by a single early fast burst of star formation and subsequent passive evolution. 
A different approach to the genetical study of galaxies emerged after cosmology provided a reliable theoretical background. Within such a background it is possible to "handle" galaxies as physical objects that evolve according to the initial and boundary conditions given by cosmology. The deductive construction of galaxies can be confronted with observations corresponding to different stages of the proto-galaxy and galaxy evolution. The breakthrough for the deductive approach was the success of the inflationary theory and the consistency of the so-called Cold Dark Matter (CDM) scenario with particle physics and observational cosmology. The main goal of these notes is to describe the ingredients, predictions, and tests of this scenario.

Galaxy evolution in action

The dramatic development of observational astronomy in the last 15 years or so opened a new window for the study of galaxy genesis: the follow up of galaxy/protogalaxy populations and their environment at different redshifts. The Deep and Ultra Deep Fields of the Hubble Spatial Telescope and other facilities allowed to discover new populations of galaxies at high redshifts, as well as to measure the evolution of global (per unit of comoving volume) quantities associated with galaxies: the cosmic star formation rate density (SFRD), the cosmic density of neutral gas, the cosmic density of metals, etc. Overall, these global quantities change significantly with $z$, in particular the SFRD as traced by the UV-luminosity at rest of galaxies [79]: since $z \sim 1.5-2$ to the present it decreased by a factor close to ten (the Universe is literally lightening off), and for higher redshifts the SFRD remains roughly constant or slightly decreases (51, 61 and the references therein). There exists indications that the SFRD at redshifts 2-4 could be approximately two times higher if considering Far Infrared/submmilimetric sources (SCUBA galaxies), where intense bursts of star formation take place in a dust-obscured phase.

Concerning populations of individual galaxies, the Deep Fields evidence a significant increase in the fraction of blue galaxies at $z \sim 1$ for the blue sequence that at these epochs look more distorted and with higher SFRs than their local counterparts. Instead, the changes observed in the red sequence are small; it seems that most red elliptical galaxies were in place long ago. At higher redshifts $(z \geq 2)$, galaxy objects with high SFRs become more and more common. The most abundant populations are:

Lyman Break Galaxies (LBG) , selected via the Lyman break at $912 \AA$ in the rest-frame. These are star-bursting galaxies (SFRs of $10-1000 \mathrm{M}_{\odot} / \mathrm{yr}$ ) with stellar masses of $10^{9}-10^{11} \mathrm{M}_{\odot}$ and moderately clustered.

Sub-millimeter (SCUBA) Galaxies, detected with sub-millimeter bolometer arrays. These are strongly star-bursting galaxies (SFRs of $\sim 1000 \mathrm{M}_{\odot} / \mathrm{yr}$ ) obscured by dust; they are strongly clustered and seem to be merging galaxies, probably precursors of ellipticals. 
Lyman $\alpha$ emitters (LAEs), selected in narrow-band studies centered in the Lyman $\alpha$ line at rest at $z>3$; strong emission Lyman $\alpha$ lines evidence phases of rapid star formation or strong gas cooling. LAEs could be young (disk?) galaxies in the early phases of rapid star formation or even before, when the gas in the halo was cooling and infalling to form the gaseous disk.

Quasars (QSOs), easily discovered by their powerful energetics; they are associated to intense activity in the nuclei of galaxies that apparently will end as spheroids; QSOs are strongly clustered and are observed up to $z \approx 6.5$.

There are many other populations of galaxies and protogalaxies at high redshifts (Luminous Red Galaxies, Damped Lyo disks, Radiogalaxies, etc.). A major challenge now is to put together all the pieces of the high-redshift puzzle to come up with a coherent picture of galaxy formation and evolution.

\section{Cosmic structure formation}

In the previous section we have learn that galaxy formation and evolution are definitively related to cosmological conditions. Cosmology provides the theoretical framework for the initial and boundary conditions of the cosmic structure formation models. At the same time, the confrontation of model predictions with astronomical observations became the most powerful testbed for cosmology. As a result of this fruitful convergence between cosmology and astronomy, there emerged the current paradigmatic scenario of cosmic structure formation and evolution of the Universe called $\Lambda$ Cold Dark Matter $(\Lambda \mathrm{CDM})$. The $\Lambda \mathrm{CDM}$ scenario integrates nicely: (1) cosmological theories (Big Bang and Inflation), (2) physical models (standard and extensions of the particle physics models), (3) astrophysical models (gravitational cosmic structure growth, hierarchical clustering, gastrophysics), and (4) phenomenology (CMBR anisotropies, non-baryonic DM, repulsive dark energy, flat geometry, galaxy properties).

Nowadays, cosmology passed from being the Cinderella of astronomy to be one of the highest precision sciences. Let us consider only the Inflation/Big Bang cosmological models with the F-R-W metric and adiabatic perturbations. The number of parameters that characterize these models is high, around 15 to be more precise. No single cosmological probe constrain all of these parameters. By using multiple data sets and probes it is possible to constrain with precision several of these parameters, many of which correlate among them (degeneracy). The main cosmological probes used for precision cosmology are the CMBR anisotropies, the type-Ia SNe and long GammaRay Bursts, the Ly $\alpha$ power spectrum, the large-scale power spectrum from galaxy surveys, the cluster of galaxies dynamics and abundances, the peculiar velocity surveys, the weak and strong lensing, the baryonic acoustic oscillation in the large-scale galaxy distribution. There is a model that is systematically consistent with most of these probes and one of the goals in the last years has 
been to improve the error bars of the parameters for this 'concordance' model. The geometry in the concordance model is flat with an energy composition dominated in $\sim 2 / 3$ by the cosmological constant $\Lambda$ (generically called Dark Energy), responsible for the current accelerated expansion of the Universe. The other $\sim 1 / 3$ is matter, but $\sim 85 \%$ of this $1 / 3$ is in form of non-baryonic DM. Table 2 presents the central values of different parameters of the $\Lambda \mathrm{CDM}$ cosmology from combined model fittings to the recent 3-year W M AP CMBR and several other cosmological probes [109] (see the WMAP website).

Table 2. Constraints to the parameters of the $\Lambda$ CDM model

\begin{tabular}{lr}
\hline Parameter & Constraint \\
\hline Total density & $\Omega=1$ \\
Dark Energy density & $\Omega_{\Lambda}=0.74$ \\
Dark Matter density & $\Omega_{D M}=0.216$ \\
Baryon Matter dens. & $\Omega_{B}=0.044$ \\
Hubble constant & $h=0.71$ \\
Age & $13.8 \mathrm{Gyr}$ \\
Power spectrum norm. & $\sigma_{8}=0.75$ \\
Power spectrum index & $n_{s}(0.002)=0.94$ \\
\hline
\end{tabular}

In the following, I will describe some of the ingredients of the $\Lambda \mathrm{CDM}$ scenario, emphasizing that most of these ingredients are well established aspects that any alternative scenario to $\Lambda \mathrm{CDM}$ should be able to explain.

\subsection{Origin of fluctuations}

The Big Bang ${ }^{4}$ is now a mature theory, based on well established observational pieces of evidence. However, the Big Bang theory has limitations. One of them is namely the origin of fluctuations that should give rise to the highly inhomogeneous structure observed today in the Universe, at scales of less than $\sim 200 \mathrm{Mpc}$. The smaller the scales, the more clustered is the matter. For example, the densities inside the central regions of galaxies, within the galaxies, cluster of galaxies, and superclusters are about $10^{11}, 10^{6}, 10^{3}$ and few times the average density of the Universe, respectively.

The General Relativity equations that describe the Universe dynamics in the Big Bang theory are for an homogeneous and isotropic fluid (Cosmological Principle); inhomogeneities are not taken into account in this theory "by definition". Instead, the concept of fluctuations is inherent to the Inflationary theory introduced in the early 80 's by A. Guth and A. Linde namely to

\footnotetext{
${ }^{4}$ It is well known that the name of 'Big Bang' is not appropriate for this theory. The key physical conditions required for an explosion are temperature and pressure gradients. These conditions contradict the Cosmological Principle of homogeneity and isotropy on which is based the 'Big Bang' theory.
} 
overcome the Big Bang limitations. According to this theory, at the energies of Grand Unification $\left(\gtrsim 10^{14} \mathrm{GeV}\right.$ or $T \gtrsim 10^{27} \mathrm{~K}$ !), the matter was in the state known in quantum field theory as vacuum. Vacuum is characterized by quantum fluctuations -temporary changes in the amount of energy in a point in space, arising from Heisenberg uncertainty principle. For a small time interval $\Delta t$, a virtual particle-antiparticle pair of energy $\Delta E$ is created (in the GU theory, the field particles are supposed to be the X- and Y-bossons), but then the pair disappears so that there is no violation of energy conservation. Time and energy are related by $\Delta E \Delta t \approx \frac{h}{2 \pi}$. The vacuum quantum fluctuations are proposed to be the seeds of present-day structures in the Universe.

How is that quantum fluctuations become density inhomogeneities? During the inflationary period, the expansion is described approximately by the de Sitter cosmology, $a \propto e^{H t}, H \equiv \dot{a} / a$ is the Hubble parameter and it is constant in this cosmology. Therefore, the proper length of any fluctuation grows as $\lambda_{p} \propto e^{H t}$. On the other hand, the proper radius of the horizon for de Sitter metric is equal to $c / H=$ const, so that initially causally connected (quantum) fluctuations become suddenly supra-horizon (classical) perturbations to the spacetime metric. After inflation, the Hubble radius grows proportional to $c t$, and at some time a given curvature perturbation cross again the horizon (becomes causally connected, $\lambda_{p}<L_{H}$ ). It becomes now a true density perturbation. The interesting aspect of the perturbation 'trip' outside the horizon is that its amplitude remains roughly constant, so that if the amplitude of the fluctuations at the time of exiting the horizon during inflation is constant (scale invariant), then their amplitude at the time of entering the horizon should be also scale invariant. In fact, the computation of classical perturbations generated by a quantum field during inflation demonstrates that the amplitude of the scalar fluctuations at the time of crossing the horizon is nearly constant, $\delta \phi_{H} \propto$ const. This can be understood on dimensional grounds: due to the Heisenberg principle $\delta \phi / \delta t \propto$ const, where $\delta t \propto H^{-1}$. Therefore, $\delta \phi_{H} \propto H$, but $H$ is roughly constant during inflation, so that $\delta \phi_{H} \propto$ const.

\subsection{Gravitational evolution of fluctuations}

The $\Lambda \mathrm{CDM}$ scenario assumes the gravitational instability paradigm: the cosmic structures in the Universe were formed as a consequence of the growth of primordial tiny fluctuations (for example seeded in the inflationary epochs) by gravitational instability in an expanding frame. The fluctuation or perturbation is characterized by its density contrast,

$$
\delta \equiv \frac{\delta \rho}{\bar{\rho}}=\frac{\rho-\bar{\rho}}{\bar{\rho}}
$$

where $\bar{\rho}$ is the average density of the Universe and $\rho$ is the perturbation density. At early epochs, $\delta<<1$ for perturbation of all scales, otherwise the homogeneity condition in the Big Bang theory is not anymore obeyed. When $\delta<<1$, the perturbation is in the linear regime and its physical size grows 
with the expansion proportional to $a(t)$. The perturbation analysis in the linear approximation shows whether a given perturbation is stable $(\delta \sim$ const or even $\rightarrow 0$ ) or unstable ( $\delta$ grows). In the latter case, when $\delta \rightarrow 1$, the linear approximation is not anymore valid, and the perturbation "separates" from the expansion, collapses, and becomes a self-gravitating structure. The gravitational evolution in the non-linear regime is complex for realistic cases and is studied with numerical $\mathrm{N}$-body simulations. Next, a pedagogical review of the linear evolution of perturbations is presented. More detailed explanations on this subject can be found in the books [72, 94, 90, 30, 77, 92.

Relevant times and scales.

The important times in the problem of linear gravitational evolution of perturbations are: (a) the epoch when inflation finished $\left(t_{\text {inf }} \approx 10^{-34} \mathrm{~s}\right.$, at this time the primordial fluctuation field is established); (b) the epoch of matterradiation equality $t_{e q}$ (corresponding to $æ \approx 1 / 3.9 \times 10^{4}\left(\Omega_{0} h^{2}\right)$, before $t_{e q}$ the dynamics of the universe is dominated by radiation density, after $t_{e q}$ dominates matter density); (c) the epoch of recombination $t_{r e c}$, when radiation decouples from baryonic matter (corresponding to $a_{r e c}=1 / 1080$, or $t_{\text {rec }} \approx 3.8 \times 10^{5} \mathrm{yr}$ for the concordance cosmology).

Scales: first of all, we need to characterize the size of the perturbation. In the linear regime, its physical size expands with the Universe: $\lambda_{p}=a(t) \lambda_{0}$, where $\lambda_{0}$ is the comoving size, by convention fixed (extrapolated) to the present epoch, $a\left(t_{0}\right)=1$. In a given (early) epoch, the size of the perturbation can be larger than the so-called Hubble radius, the typical radius over which physical processes operate coherently (there is causal connection): $L_{H} \equiv(a / \dot{a})^{-1}=H^{-1}=n^{-1} c t$. For the radiation or matter dominated cases, $a(t) \propto t^{n}$, with $n=1 / 2$ and $n=2 / 3$, respectively, that is $n<1$. Therefore, $L_{H}$ grows faster than $\lambda_{p}$ and at a given "crossing" time $t_{\text {cross }}, \lambda_{p}<L_{H}$. Thus, the perturbation is supra-horizon sized at epochs $t<t_{\text {cross }}$ and sub-horizon sized at $t>t_{\text {cross }}$. Notice that if $n>1$, then at some time the perturbation "exits" the Hubble radius. This is what happens in the inflationary epoch, when $a(t) \propto e^{t}$ : causally-connected fluctuations of any size are are suddenly "taken out" outside the Hubble radius becoming causally disconnected.

For convenience, in some cases it is better to use masses instead of sizes. Since in the linear regime $\delta<<1(\rho \approx \bar{\rho})$, then $M \approx \rho_{M}(a) \ell^{3}$, where $\ell$ is the size of a given region of the Universe with average matter density $\rho_{M}$. The mass of the perturbation, $M_{p}$, is invariant.

\section{Supra-horizon sized perturbations.}

In this case, causal, microphysical processes are not possible, so that it does not matter what perturbations are made of (baryons, radiation, dark matter, etc.); they are in general just perturbations to the metric. To study the gravitational growth of metric perturbations, a General Relativistic analysis is necessary. A major issue in carrying out this program is that the metric 
perturbation is not a gauge invariant quantity. See e.g., 72 for an outline of how E. Lifshitz resolved brilliantly this difficult problem in 1946. The result is quite simple and it shows that the amplitude of metric perturbations outside the horizon grows kinematically at different rates, depending on the dominant component in the expansion dynamics. For the critical cosmological model (at early epochs all models approach this case), the growing modes of metric perturbations according to what dominates the background are:

$$
\begin{aligned}
& \delta_{m,+} \propto a(t) \propto t^{2 / 3}, \ldots \ldots \ldots \ldots \ldots \ldots . . . m a t t e r \\
& \delta_{m,+} \propto a(t)^{2} \propto t, \ldots \ldots \ldots \ldots \ldots . . . . . \text { radiation } \\
& \left.\delta_{m,+} \propto a(t)^{-2} \propto e^{-2 H t}, . . \Lambda \text { (deSitter }\right)
\end{aligned}
$$

Sub-horizon sized perturbations.

Once perturbations are causally connected, microphysical processes are switched on (pressure, viscosity, radiative transport, etc.) and the gravitational evolution of the perturbation depends on what it is made of. Now, we deal with true density perturbations. For them applies the classical perturbation analysis for a fluid, originally introduced by J. Jeans in 1902, in the context of the problem of star formation in the ISM. But unlike in the ISM, in the cosmological context the fluid is expanding. What can prevent the perturbation amplitude from growing gravitationally? The answer is pressure support. If the fluid pressure gradient can re-adjust itself in a timescale $t_{\text {press }}$ smaller than the gravitational collapse timescale, $t_{\text {grav }}$, then pressure prevents the gravitational growth of $\delta$. Thus, the condition for gravitational instability is:

$$
t_{\text {grav }} \approx \frac{1}{(G \rho)^{1 / 2}}<t_{\text {press }} \approx \frac{\lambda_{p}}{v},
$$

where $\rho$ is the density of the component that is most gravitationally dominant in the Universe, and $v$ is the sound speed (collisional fluid) or velocity dispersion (collisionless fluid) of the perturbed component. In other words, if the perturbation scale is larger than a critical scale $\lambda_{J} \sim v(G \rho)^{-1 / 2}$, then pressure loses, gravity wins.

The perturbation analysis applied to the hydrodynamical equations of a fluid at rest shows that $\delta$ grows exponentially with time for perturbations obeying the Jeans instability criterion $\lambda_{p}>\lambda_{J}$, where the exact value of $\lambda_{J}$ is $v(\pi / G \rho)^{1 / 2}$. If $\lambda_{p}<\lambda_{J}$, then the perturbations are described by stable gravito-acustic oscillations. The situation is conceptually similar for perturbations in an expanding cosmological fluid, but the growth of $\delta$ in the unstable regime is algebraical instead of exponential. Thus, the cosmic structure formation process is relatively slow. Indeed, the typical epochs of galaxy and cluster of galaxies formation are at redshifts $z \sim 1-5$ (ages of $\sim 1.2-6$ Gyrs) and $z<1$ (ages larger than 6 Gyrs), respectively. 
Baryonic matter. The Jeans instability analysis for a relativistic (plasma) fluid of baryons ideally coupled to radiation and expanding at the rate $H=$ $\dot{a} / a$ shows that there is an instability critical scale $\lambda_{J}=v(3 \pi / 8 G \rho)^{1 / 2}$, where the sound speed for adiabatic perturbations is $v=p / \rho=c / \sqrt{3}$; the latter equality is due to pressure radiation. At the epoch when radiation dominates, $\rho=\rho_{r} \propto a^{-4}$ and then $\lambda_{J} \propto a^{2} \propto c t$. It is not surprising that at this epoch $\lambda_{J}$ approximates the Hubble scale $L_{H} \propto c t$ (it is in fact $\sim 3$ times larger). Thus, perturbations that might collapse gravitationally are in fact outside the horizon, and those that already entered the horizon, have scales smaller than $\lambda_{J}$ : they are stable gravito-acoustic oscillations. When matter dominates, $\rho=\rho_{M} \propto a^{-3}$, and $a \propto t^{2 / 3}$. Therefore, $\lambda_{J} \propto a \propto t^{2 / 3} \underset{\sim}{\sim} L_{H}$, but still radiation is coupled to baryons, so that radiation pressure is dominant and $\lambda_{J}$ remains large. However, when radiation decouples from baryons at $t_{r e c}$, the pressure support drops dramatically by a factor of $P_{r} / P_{b} \propto n_{r} T / n_{b} T \approx 10^{8}$ ! Now, the Jeans analysis for a gas mix of $\mathrm{H}$ and He at temperature $T_{\text {rec }} \approx 4000 \mathrm{~K}$ shows that baryonic clouds with masses $\gtrsim 10^{6} \mathrm{M}_{\odot}$ can collapse gravitationally, i.e. all masses of cosmological interest. But this is literally too "ideal" to be true.

The problem is that as the Universe expands, radiation cools $\left(T_{r}=T_{0} a^{-1}\right)$ and the photon-baryon fluid becomes less and less perfect: the mean free path for scattering of photons by electrons (which at the same time are coupled electrostatically to the protons) increases. Therefore, photons can diffuse out of the bigger and bigger density perturbations as the photon mean free path increases. If perturbations are in the gravito-acoustic oscillatory regime, then the oscillations are damped out and the perturbations disappear. The "ironing out" of perturbations continues until the epoch of recombination. In a pioneering work, J. Silk [104] carried out a perturbation analysis of a relativistic cosmological fluid taking into account radiative transfer in the diffusion approximation. He showed that all photon-baryon perturbations of masses smaller than $M_{S}$ are "ironed out" until $t_{r e c}$ by the (Silk) damping process. The first crisis in galaxy formation theory emerged: calculations showed that $M_{S}$ is of the order of $10^{13}-10^{14} \mathrm{M}_{\odot} \mathrm{h}^{-1}$ ! If somebody (god, inflation, ...) seeded primordial fluctuations in the Universe, by Silk damping all galaxysized perturbation are "ironed out". ${ }^{5}$

Non-baryonic matter. The gravito-acoustic oscillations and their damping by photon diffusion refer to baryons. What happens for a fluid of non-baryonic DM? After all, astronomers, since Zwicky in the 1930s, find routinely pieces

\footnotetext{
${ }^{5}$ In the 1970s Y. Zel'dovich and collaborators worked out a scenario of galaxy formation starting from very large perturbations, those that were not affected by Silk damping. In this elegant scenario, the large-scale perturbations, considered in a first approximation as ellipsoids, collapse most rapidly along their shortest axis, forming flattened structures ("pancakes"), which then fragment into galaxies by gravitational or thermal instabilities. In this 'top-down' scenario, to obtain galaxies in place at $z \sim 1$, the amplitude of the large perturbations at recombination should be $\geq 3 \times 10^{-3}$. Observations of the CMBR anisotropies showed that the amplitudes are 1-2 order of magnitudes smaller than those required.
} 


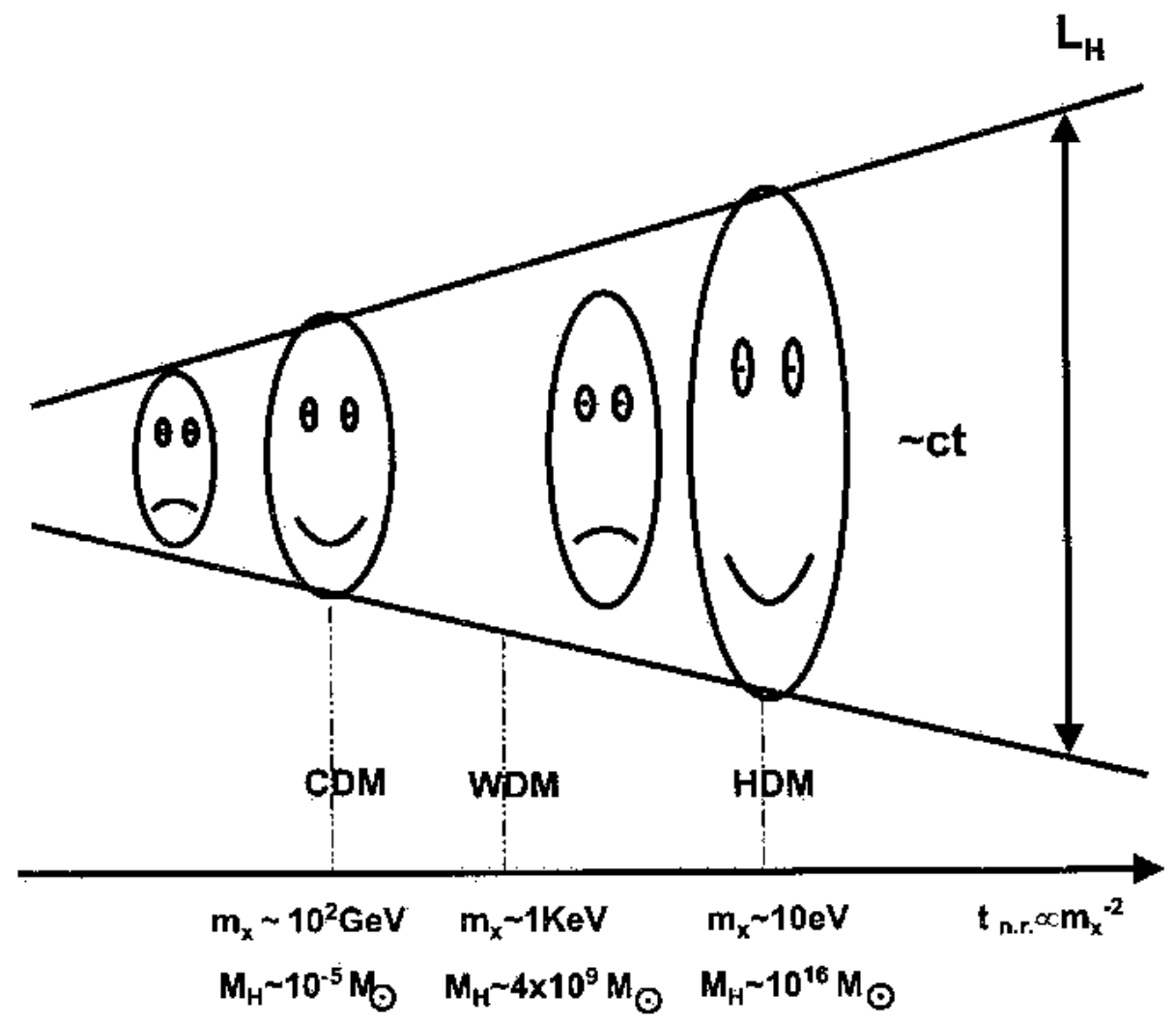

Fig. 4. Free-streaming damping kills perturbations of sizes roughly smaller than the horizon length if they are made of relativistic particles. The epoch $t_{n . r}$. when thermal-coupled particles become non-relativistic is inverse proportional to the square of the particle mass $m_{X}$. Typical particle masses of CDM, WDM and HDM are given together with the corresponding horizon (filtering) masses.

of evidence for the presence of large amounts of DM in the Universe. As DM is assumed to be collisionless and not interacting electromagnetically, then the radiative or thermal pressure supports are not important for linear DM perturbations. However, DM perturbations can be damped out by free streaming if the particles are relativistic: the geodesic motion of the particles at the speed of light will iron out any perturbation smaller than a scale close to the particle horizon radius, because the particles can freely propagate from an overdense region to an underdense region. Once the particles cool and become non relativistic, free streaming is not anymore important. A particle of mass $m_{X}$ and temperature $T_{X}$ becomes non relativistic when $k_{B} T_{X} \sim m_{X} c^{2}$. Since $T_{X} \propto a^{-1}$, and $a \propto t^{1 / 2}$ when radiation dominates, one then finds that the epoch when a thermal-relic particle becomes non relativistic is $t_{n r} \propto m_{X}^{-2}$. 
The more massive the DM particle, the earlier it becomes non relativistic, and the smaller are therefore the perturbations damped out by free streaming (those smaller than $\sim c t$; see Fig. (4). According to the epoch when a given thermal DM particle species becomes non relativistic, DM is called Cold Dark Matter (CDM, very early), Warm Dark Matter (WDM, early) and Hot Dark Matter (HDM, late) ${ }^{6}$.

The only non-baryonic particles confirmed experimentally are (light) neutrinos (HDM). For neutrinos of masses $\sim 1-10 \mathrm{eV}$, free streaming attains to iron out perturbations of scales as large as massive clusters and superclusters of galaxies (see Fig. 4). Thus, HDM suffers the same problem of baryonic matter concerning galaxy formation ${ }^{7}$. At the other extreme is $\mathrm{CDM}$, in which case survive free streaming practically all scales of cosmological interest. This makes CDM appealing to galaxy formation theory. In the minimal CDM model, it is assumed that perturbations of all scales survive, and that CDM particles are collisionless (they do not self-interact). Thus, if CDM dominates, then the first step in galaxy formation study is reduced to the calculation of the linear and non-linear gravitational evolution of collisionless CDM perturbations. Galaxies are expected to form in the centers of collapsed CDM structures, called halos, from the baryonic gas, first trapped in the gravitational potential of these halos, and second, cooled by radiative (and turbulence) processes (see $\S 5$ ).

The CDM perturbations are free of any physical damping processes and in principle their amplitudes may grow by gravitational instability. However, when radiation dominates, the perturbation growth is stagnated by expansion. The gravitational instability timescale for sub-horizon linear CDM perturbations is $t_{\text {grav }} \sim\left(G \rho_{D M}\right)^{-2}$, while the expansion (Hubble) timescale is given by $t_{\text {exp }} \sim(G \bar{\rho})^{-2}$. When radiation dominates, $\bar{\rho} \approx \rho_{r}$ and $\rho_{r}>>\rho_{M}$. Therefore $t_{\text {exp }}<<t_{\text {grav }}$, that is, expansion is faster than the gravitational shrinking.

Fig. 5 resumes the evolution of primordial perturbations. Instead of spatial scales, in Fig. 5 are shown masses, which are invariant for the perturbations. We highlight the following conclusions from this plot: (1) Photonbaryon perturbations of masses $<M_{S}$ are washed out $\left(\delta_{B} \rightarrow 0\right)$ as long as baryon matter is coupled to radiation. (2) The amplitude of CDM perturbations that enter the horizon before $t_{e q}$ is "freezed-out" $\left(\delta_{D M} \propto \mathrm{const}\right)$ as long as radiation dominates; these are perturbations of masses smaller than $M_{H, e q} \approx 10^{13}\left(\Omega_{M} h^{2}\right)^{-2} \mathrm{M}_{\odot}$, namely galaxy scales. (3) The baryons are trapped gravitationally by CDM perturbations, and within a factor of two in $z$, baryon perturbations attain amplitudes half that of $\delta_{D M}$. For WDM

\footnotetext{
6 The reference to "early" and "late" is given by the epoch and the corresponding radiation temperature when the largest galaxy-sized perturbations $(M \sim$ $\left.10^{13} \mathrm{M}_{\odot}\right)$ enter the horizon: $a_{\text {gal }} \sim æ \approx 1 / 3.9 \times 10^{4}\left(\Omega_{0} h^{2}\right)$ and $T_{r} \sim 1 \mathrm{KeV}$.

7 Neutrinos exist and have masses larger than $0.05 \mathrm{eV}$ according to determinations based on solar neutrino oscillations. Therefore, neutrinos contribute to the matter density in the Universe. Cosmological observations set a limit: $\Omega_{\nu} h^{2}<0.0076$, otherwise too much structure is erased.
} 
or HDM perturbations, the free-streaming damping introduces a mass scale

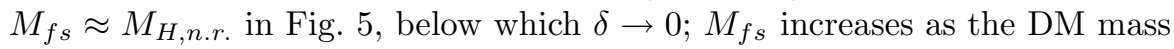
particle decreases (Fig. (4).

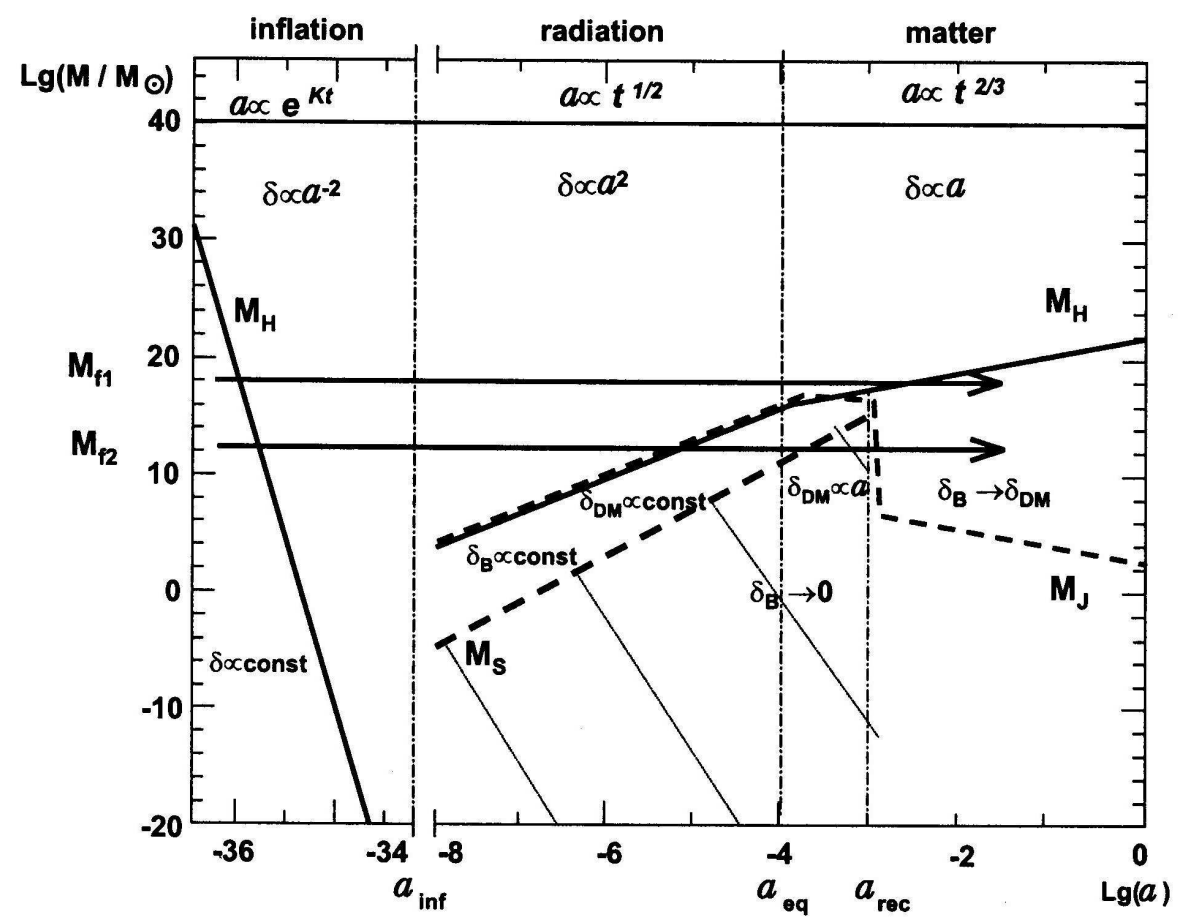

Fig. 5. Different evolutive regimes of perturbations $\delta$. The suffixes "B" and "DM" are for baryon-photon and DM perturbations, respectively. The evolution of the horizon, Jeans and Silk masses $\left(M_{H}, M_{J}\right.$, and $\left.M_{S}\right)$ are showed. $M_{f 1}$ and $M_{f 2}$ are the masses of two perturbations. See text for explanations.

The processed power spectrum of perturbations. The exact solution to the problem of linear evolution of cosmological perturbations is much more complex than the conceptual aspects described above. Starting from a primordial fluctuation field, the perturbation analysis should be applied to a cosmological mix of baryons, radiation, neutrinos, and other non-baryonic dark matter components (e.g., CDM), at sub- and supra-horizon scales (the fluid assumption is relaxed). Then, coupled relativistic hydrodynamic and Boltzmann equations in a general relativity context have to be solved taking into account radiative and dissipative processes. The outcome of these complex calculations is the full description of the processed fluctuation field at the recombination epoch (when fluctuations at almost all scales are still in the linear regime). The goal is double and of crucial relevance in cosmology and astrophysics: 
1) to predict the physical and statistical properties of CMBR anisotropies, which can be then compared with observations, and 2) to provide the initial conditions for calculating the non-linear regime of cosmic structure formation and evolution. Fortunately, there are now several public friendly-to-use codes that numerically solve the cosmological linear perturbation equations (e.g., CMBFast and $\mathrm{CAMB}^{8}$ ).

The description of the density fluctuation field is statistical. As any random field, it is convenient to study perturbations in the Fourier space. The Fourier expansion of $\delta(\mathbf{x})$ is:

$$
\begin{array}{r}
\delta(\mathbf{x})=\frac{V}{(2 \pi)^{3}} \int \delta_{k} e^{-i \mathbf{k x}} d^{3} k, \\
\delta_{k}=V^{-1} \int \delta(\mathbf{x}) e^{i \mathbf{k x}} d^{3} x
\end{array}
$$

The Fourier modes $\delta_{k}$ evolve independently while the perturbations are in the linear regime, so that the perturbation analysis can be applied to this quantity. For a Gaussian random field, any statistical quantity of interest can be specified in terms of the power spectrum $P(k) \equiv\left|\delta_{k}\right|^{2}$, which measures the amplitude of the fluctuations at a given scale $k^{9}$. Thus, from the linear perturbation analysis we may follow the evolution of $P(k)$. A more intuitive quantity than $P(k)$ is the mass variance $\sigma_{M}^{2} \equiv\left\langle(\delta M / M)_{R}^{2}\right\rangle$ of the fluctuation field. The physical meaning of $\sigma_{M}$ is that of an rms density contrast on a given sphere of radius $R$ associated to the mass $M=\rho V_{W}(R)$, where $W(R)$ is a window (smoothing) function. The mass variance is related to $P(k)$. By assuming a power law power spectrum, $P(k) \propto k^{n}$, it is easy to show that

$$
\begin{aligned}
\sigma_{M} \propto R^{-(3+n)} \propto M^{-(3+n) / 3} & =M^{-2 \alpha} \\
\alpha & =\frac{3+n}{6},
\end{aligned}
$$

for $4<n<-3$ using a Gaussian window function. The question is: How the scaling law of perturbations, $\sigma_{M}$, evolves starting from an initial $\left(\sigma_{M}\right)_{i}$ ?

In the early 1970s, Harrison and Zel'dovich independently asked themselves about the functionality of $\sigma_{M}$ (or the density contrast) at the time adiabatic perturbations cross the horizon, that is, if $\left(\sigma_{M}\right)_{H} \propto M^{\alpha_{H}}$, then what is the value of $\alpha_{H}$ ? These authors concluded that $-0.1 \leq \alpha_{H} \leq 0.2$, i.e.

\footnotetext{
${ }^{8}$ http://www.cmbfast.org and http://camb.info/

9 The phases of the Fourier modes in the Gaussian case are uncorrelated. Gaussianity is the simplest assumption for the primordial fluctuation field statistics and it seems to be consistent with some variants of inflation. However, there are other variants that predict non-Gaussian fluctuations (for a recent review on this subject see e.g. [8), and the observational determination of the primordial fluctuation statistics is currently an active field of investigation. The properties of cosmic structures depend on the assumption about the primordial statistics, not only at large scales but also at galaxy scales; see for a review and new results 4 .
} 
$\alpha_{H} \approx 0\left(n_{H} \approx-3\right)$. If $\alpha_{H}>>0\left(n_{H}>>-3\right)$, then $\sigma_{M} \rightarrow \infty$ for $M \rightarrow 0$; this means that for a given small mass scale $M$, the mass density of the perturbation at the time of becoming causally connected can correspond to the one of a (primordial) black hole. Hawking evaporation of black holes put a constraint on $M_{B H, \text { prim }} \lesssim 10^{15} \mathrm{~g}$, which corresponds to $\alpha_{H} \leq 0.2$, otherwise the $\gamma$-ray background radiation would be more intense than that observed. If $\alpha_{H}<<0$ $\left(n_{H}<<-3\right)$, then larger scales would be denser than the small ones, contrary to what is observed. The scale-invariant Harrison-Zel'dovich power spectrum, $P_{H}(k) \propto k^{-3}$, is for perturbations at the time of entering the horizon. How should the primordial power spectrum $P_{i}(k)=A k_{i}^{n}$ or $\left(\sigma_{M}\right)_{i}=B M^{-\alpha_{i}}$ (defined at some fixed initial time) be to produce such scale invariance? Since $t_{i}$ until the horizon crossing time $t_{\text {cross }}(M)$ for a given perturbation of mass $M$, $\sigma_{M}(t)$ evolves as $a(t)^{2}$ (supra-horizon regime in the radiation era). At $t_{\text {cross }}$, the horizon mass $M_{H}$ is equal by definition to $M$. We have seen that $M_{H} \propto a^{3}$ (radiation dominion), so that $a_{\text {cross }} \propto M_{H}^{1 / 3}=M^{1 / 3}$. Therefore,

$$
\sigma_{M}\left(t_{\text {cross }}\right) \propto\left(\sigma_{M}\right)_{i}\left(a_{\text {cross }} / a_{i}\right)^{2} \propto M^{-\alpha_{i}} M^{2 / 3},
$$

i.e. $\alpha_{H}=2 / 3-\alpha_{i}$ or $n_{H}=n_{i}-4$. A similar result is obtained if the perturbation enters the horizon during the matter dominion era. From this analysis one concludes that for the perturbations to be scale invariant at horizon crossing $\left(\alpha_{H}=0\right.$ or $\left.n_{H}=-3\right)$, the primordial (initial) power spectrum should be $P_{i}(k)=A k^{1}$ or $\left(\sigma_{M}\right)_{i} \propto M^{-2 / 3} \propto \lambda_{0}^{-2}$ (i.e. $n_{i}=1$ and $\alpha=2 / 3 ; A$ is a normalization constant). Does inflation predict such power spectrum? We have seen that, according to the quantum field theory and assuming that $H=$ const during inflation, the fluctuation amplitude is scale invariant at the time to exit the horizon, $\delta_{H} \sim$ const. On the other hand, we have seen that supra-horizon curvature perturbations during a de Sitter period evolve as $\delta \propto a^{-2}$ (eq. 4). Therefore, at the end of inflation we have that $\delta_{i n f}=\delta_{H}\left(\lambda_{0}\right)\left(a_{i n f} / a_{H}\right)^{-2}$. The proper size of the fluctuation when crossing the horizon is $\lambda_{p}=a_{H} \lambda_{0} \approx H^{-1}$; therefore, $a_{H} \approx 1 /\left(\lambda_{0} H\right)$. Replacing now this expression in the equation for $\delta_{\text {inf }}$ we get that:

$$
\delta_{i n f} \approx \delta_{H}\left(\lambda_{0}\right)\left(a_{i n f} \lambda_{0} H\right)^{-2} \propto \lambda_{0}^{-2} \propto M^{-2 / 3},
$$

if $\delta_{H} \sim$ const. Thus, inflation predicts $\alpha_{i}$ nearly equal to $2 / 3\left(n_{i} \approx 1\right)$ ! Recent results from the analysis of CMBR anisotropies by the WMAP satellite 109 seem to show that $n_{i}$ is slightly smaller than 1 or that $n_{i}$ changes with the scale (running power-spectrum index). This is in more agreement with several inflationary models, where $H$ actually slightly vary with time introducing some scale dependence in $\delta_{H}$.

The perturbation analysis, whose bases were presented in $\S 3.2$ and resumed in Fig. [5] show us that $\sigma_{M}$ grows (kinematically) while perturbations are in the supra-horizon regime. Once perturbations enter the horizon (first the smaller ones), if they are made of CDM, then the gravitational growth is "freezed out" whilst radiation dominates (stangexpantion). As shown schematically 


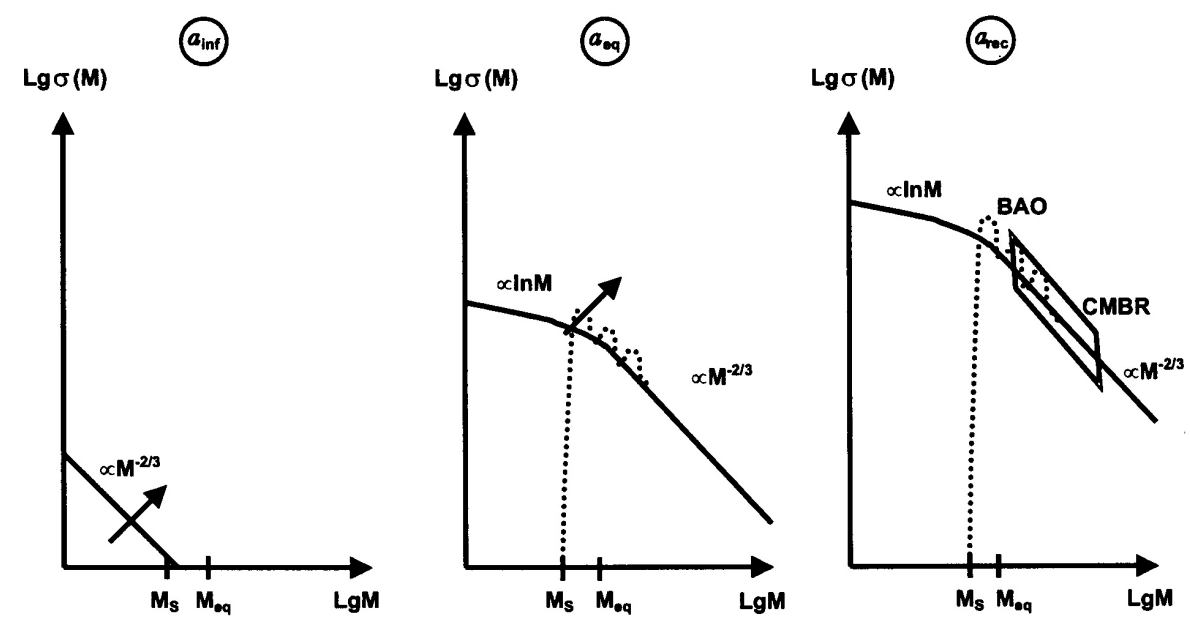

Fig. 6. Linear evolution of the perturbation mass variance $\sigma_{M}$. The perturbation amplitude in the supra-horizon regime grow kinematically. DM perturbations (solid curve) that cross the horizon during the radiation dominion, freeze-out their grow due to stangexpantion, producing a flattening in the scaling law $\sigma_{M}$ for all scales smaller than the corresponding to the horizon at the equality epoch (galaxy scales). Baryon-photon perturbations smaller than the Silk mass $M_{S}$ are damped out (dotted curve) and those larger than $M_{S}$ but smaller than the horizon mass at recombination are oscillating (Baryonic Acoustic Oscillation, BAO).

in Fig. 6] this "flattens" the variance $\sigma_{M}$ at scales smaller than $M_{H, e q}$; in fact, $\sigma_{M} \propto \ln (M)$ at these scales, corresponding to galaxies! After $t_{e q}$ the CDM variance (or power spectrum) grows at the same rate at all scales. If perturbations are made out of baryons, then for scales smaller than $M_{S}$, the gravito-acoustic oscillations are damped out, while for scales close to the Hubble radius at recombination, these oscillations are present. The "final" processed mass variance or power spectrum is defined at the recombination epoch. For example, the power spectrum is expressed as:

$$
P_{r e c}(k)=A k^{n_{i}} \times\left(D\left(t_{r e c}\right) / D\left(t_{i}\right)\right)^{2} \times T^{2}(k),
$$

where the first term is the initial power spectrum $P_{i}(k)$; the second one is how much the fluctuation amplitude has grown in the linear regime $(D(t)$ is the so-called linear growth factor), and the third one is a transfer function that encapsulates the different damping and freezing out processes able to deform the initial power spectrum shape. At large scales, $T^{2}(k)=1$, i.e. the primordial shape is conserved (see Fig. [6).

Besides the mass power spectrum, it is possible to calculate the angular power spectrum of temperature fluctuation in the CMBR. This spectrum consists basically of 2 ranges divided by a critical angular scales: the angle $\theta_{h}$ corresponding to the horizon scale at the epoch of recombination 
$\left(\left(L_{H}\right)_{\text {rec }} \approx 200\left(\Omega h^{2}\right)^{-1 / 2} \mathrm{Mpc}\right.$, comoving $)$. For scales grander than $\theta_{h}$ the spectrum is featureless and corresponds to the scale-invariant supra-horizon Sachs-Wolfe fluctuations. For scales smaller than $\theta_{h}$, the sub-horizon fluctuations are dominated by the Doppler scattering (produced by the gravitoacoustic oscillations) with a series of decreasing in amplitude peaks; the position (angle) of the first Doppler peak depends strongly on $\Omega$, i.e. on the geometry of the Universe. In the last 15 years, high-technology experiments as COBE, Boomerang, WMAP provided valuable information (in particular the latter one) on CMBR anisotropies. The results of this exciting branch of astronomy (called sometimes anisotronomy) were of paramount importance for astronomy and cosmology (see for a review [62 and the W. Hu website ${ }^{10}$ ).

Just to highlight some of the key results of CMBR studies, let us mention the next ones: 1) detailed predictions of the $\Lambda \mathrm{CDM}$ scenario concerning the linear evolution of perturbations were accurately proved, 2) several cosmological parameters as the geometry of the Universe, the baryonic fraction $\Omega_{B}$, and the index of the primordial power spectrum, were determined with high precision (see the actualized, recently delivered results from the 3 year analysis of WMAP in [109), 3) by studying the polarization maps of the CMBR it was possible to infer the epoch when the Universe started to be significantly reionized by the formation of first stars, 4) the amplitude (normalization) of the primordial fluctuation power spectrum was accurately measured. The latter is crucial for further calculating the non-linear regime of cosmic structure formation. I should emphasize that while the shape of the power spectrum is predicted and well understood within the context of the $\Lambda \mathrm{CDM}$ model, the situation is fuzzy concerning the power spectrum normalization. We have a phenomenological value for $A$ but not a theoretical prediction.

\section{The dark side of galaxy formation and evolution}

A great triumph of the $\Lambda \mathrm{CDM}$ scenario was the overall consistency found between predicted and observed CMBR anisotropies generated at the recombination epoch. In this scenario, the gravitational evolution of CDM perturbations is the driver of cosmic structure formation. At scales much larger than galaxies, (i) mass density perturbations are still in the (quasi)linear regime, following the scaling law of primordial fluctuations, and (ii) the dissipative physics of baryons does not affect significantly the matter distribution. Thus, the large-scale structure (LSS) of the Universe is determined basically by DM perturbations yet in their (quasi)linear regime. At smaller scales, nonlinearity strongly affects the primordial scaling law and, moreover, the dissipative physics of baryons "distorts" the original DM distribution, particularly inside galaxy-sized DM halos. However, DM in any case provides the original "mold" where gas dynamics processes take place.

${ }^{10}$ http://background.uchicago.edu/ whu/physics/physics.html 
The $\Lambda$ CDM scenario describes successfully the observed LSS of the Universe (for reviews see e.g., 49 ,58, and for some recent observational results see e.g. [115, 102, 109]). The observed filamentary structure can be explained as a natural consequence of the CDM gravitational instability occurring preferentially in the shortest axis of 3D and 2D protostructures (the Zel'dovich panckakes). The clustering of matter in space, traced mainly by galaxies, is also well explained by the clustering properties of CDM. At scales $r$ much larger than typical galaxy sizes, the galaxy 2-point correlation function $\xi_{\text {gal }}(r)$ (a measure of the average clustering strength on spheres of radius $r$ ) agrees rather well with $\xi_{C D M}(r)$. Current large statistical galaxy surveys as SDSS and 2dFGRS, allow now to measure the redshift-space 2-point correlation function at large scales with unprecedented accuracy, to the point that weak "bumps" associated with the baryon acoustic oscillations at the recombination epoch begin to be detected [41]. At small scales $\left(\stackrel{\sim}{\sim} 3 \mathrm{Mpch}^{-1}\right), \xi_{\text {gal }}(r)$ departs from the predicted pure $\xi_{C D M}(r)$ due to the emergence of two processes: (i) the strong non-linear evolution that small scales underwent, and (ii) the complexity of the baryon processes related to galaxy formation. The difference between $\xi_{\text {gal }}(r)$ and $\xi_{C D M}(r)$ is parametrized through one "ignorance" parameter, $b$, called bias, $\xi_{\text {gal }}(r)=b \xi_{C D M}(r)$. Below, some basic ideas and results related to the former processes will be described. The baryonic process will be sketched in the next Section.

\subsection{Nonlinear clustering evolution}

The scaling law of the processed $\Lambda \mathrm{CDM}$ perturbations, is such that $\sigma_{M}$ at galaxy-halo scales decreases slightly with mass (logarithmically) and for larger scales, decreases as a power law (see Fig. [6). Because the perturbations of higher amplitudes collapse first, the first structures to form in the $\Lambda \mathrm{CDM}$ scenario are typically the smallest ones. Larger structures assemble from the smaller ones in a process called hierarchical clustering or bottom-up mass assembling. It is interesting to note that the concept of hierarchical clustering was introduced several years before the CDM paradigm emerged. Two seminal papers settled the basis for the current theory of galaxy formation: Press \& Schechter 1974 98 and White \& Rees 1979 [131]. In the latter it was proposed that "the smaller-scale virialized [dark] systems merge into an amorphous whole when they are incorporated in a larger bound cluster. Residual gas in the resulting potential wells cools and acquires sufficient concentration to self-gravitate, forming luminous galaxies up to a limiting size".

The Press \& Schechter (P-S) formalism was developed to calculate the mass function (per unit of comoving volume) of halos at a given epoch, $n(M, z)$. The starting point is a Gaussian density field filtered (smoothed) at different scales corresponding to different masses, the mass variance $\sigma_{M}$ being the characterization of this filtering process. A collapsed halo is identified when the evolving density contrast of the region of mass $M, \delta_{M}(z)$, 
attains a critical value, $\delta_{c}$, given by the spherical top-hat collapse model ${ }^{11}$. This way, the Gaussian probability distribution for $\delta_{M}$ is used to calculate the mass distribution of objects collapsed at the epoch $z$. The P-S formalism assumes implicitly that the only objects to be counted as collapsed halos at a given epoch are those with $\delta_{M}(z)=\delta_{c}$. For a mass variance decreasing with mass, as is the case for CDM models, this implies a "hierarchical" evolution of $n(M, z)$ : as $z$ decreases, less massive collapsed objects disappear in favor of more massive ones (see Fig. (8). The original P-S formalism had an error of 2 in the sense that integrating $n(M, z)$ half of the mass is lost. The authors multiplied $n(M, z)$ by 2 , argumenting that the objects duplicate their masses by accretion from the sub-dense regions. The problem of the factor of 2 in the P-S analysis was partially solved using an excursion set statistical approach [17, 73.

To get an idea of the typical formation epochs of CDM halos, the spherical collapse model can be used. According to this model, the density contrast of given overdense region, $\delta$, grows with $z$ proportional to the growing factor, $D(z)$, until it reaches a critical value, $\delta_{c}$, after which the perturbation is supposed to collapse and virialize ${ }^{12}$. at redshift $z_{\text {col }}$ (for example see 90]):

$$
\delta\left(z_{\mathrm{col}}\right) \equiv \delta_{0} D\left(z_{\mathrm{col}}\right)=\delta_{c, 0} .
$$

The convention is to fix all the quantities to their linearly extrapolated values at the present epoch (indicated by the subscript " 0 ") in such a way that $D(z=$ $0) \equiv D_{0}=1$. Within this convention, for an Einstein-de Sitter cosmology, $\delta_{c, 0}=1.686$, while for the $\Lambda \mathrm{CDM}$ cosmology, $\delta_{c, 0}=1.686 \Omega_{M, 0}^{0.0055}$, and the growing factor is given by

$$
D(z)=\frac{g(z)}{g\left(z_{0}\right)(1+z)}
$$

\footnotetext{
${ }^{11}$ The spherical top-hat model refers to the exact calculation of the collapse of a uniform spherical density perturbation in an otherwise uniform Universe; the dynamics of such a region is the same of a closed Universe. The solution of the equations of motion shows that the perturbation at the beginning expands as the background Universe (proportional to $a$ ), then it reaches a maximum expansion (size) in a time $t_{\max }$, and since that moment the perturbation separates of the expanding background, collapsing in a time $t_{c o l}=2 t_{\max }$.

12 The mathematical solution gives that the spherical perturbed region collapses into a point (a black hole) after reaching its maximum expansion. However, real perturbations are lumpy and the particle orbits are not perfectly radial. In this situation, during the collapse the structure comes to a dynamical equilibrium under the influence of large scale gravitational potential gradients, a process named by the oxymoron "violent relaxation" (see e.g. [14]); this is a typical collective phenomenon. The end result is a system that satisfies the virial theorem: for a self-gravitating system this means that the internal kinetic energy is half the (negative) gravitational potential energy. Gravity is supported by the velocity dispersion of particles or lumps. The collapse factor is roughly $1 / 2$, i.e. the typical virial radius $R_{v}$ of the collapsed structure is $\approx 0.5$ the radius of the perturbation at its maximum expansion.
} 
where a good approximation for $g(z)$ is [23]:

$$
g(z) \simeq \frac{5}{2}\left[\Omega_{M}^{\frac{4}{7}}-\Omega_{\Lambda}+\left(1+\frac{\Omega_{M}}{2}\right)\left(1+\frac{\Omega_{\Lambda}}{70}\right)\right]^{-1},
$$

and where $\Omega_{M}=\Omega_{M, 0}(1+z)^{3} / E^{2}(z), \Omega_{\Lambda}=\Omega_{\Lambda} / E^{2}(z)$, with $E^{2}(z)=\Omega_{\Lambda}+$ $\Omega_{M, 0}(1+z)^{3}$. For the Einstein-de Sitter model, $D(z)=(1+z)$. We need now to connect the top-hat sphere results to a perturbation of mass $M$. The processed perturbation field, fixed at the present epoch, is characterized by the mass variance $\sigma_{M}$ and we may assume that $\delta_{0}=\nu \sigma_{M}$, where $\delta_{0}$ is $\delta$ linearly extrapolated to $z=0$, and $\nu$ is the peak height. For average perturbations, $\nu=$ 1 , while for rare, high-density perturbations, from which the first structures arose, $\nu>>1$. By introducing $\delta_{0}=\nu \sigma_{M}$ into eq. (11) one may infer $z_{c o l}$ for a given mass. Fig. 7 shows the typical $z_{c o l}$ of $1 \sigma, 2 \sigma$, and $3 \sigma$ halos. The collapse of galaxy-sized $1 \sigma$ halos occurs within a relatively small range of redshifts. This is a direct consequence of the "flattening" suffered by $\sigma_{M}$ during radiation-dominated era due to stangexpansion (see §3.2). Therefore, in a $\Lambda \mathrm{CDM}$ Universe it is not expected to observe a significant population of galaxies at $z \geq 5$.

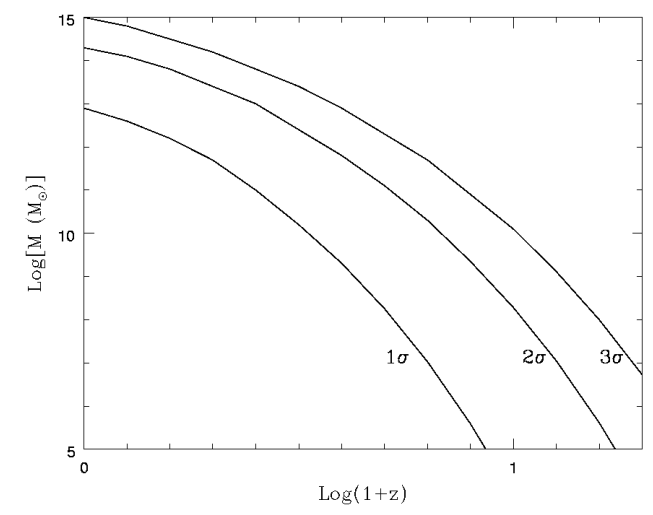

Fig. 7. Collapse redshifts of spherical top-hat $1 \sigma, 2 \sigma$ and $3 \sigma$ perturbations in a $\Lambda \mathrm{CDM}$ cosmology with $\sigma_{8}=0.9$. Note that galaxy-sized $\left(M \sim 10^{8}-10^{13} \mathrm{M}_{\odot}\right)$ $1 \sigma$ halos collapse in a redshift range, from $z \sim 3.5$ to $z=0$, respectively; the corresponding ages are from $\sim 1.9$ to $13.8 \mathrm{Gyr}$, respectively.

The problem of cosmological gravitational clustering is very complex due to non-linearity, lack of symmetry and large dynamical range. Analytical and semi-analytical approaches provide illuminating results but numerical $\mathrm{N}$-body simulations are necessary to tackle all the aspects of this problem. In the last 20 years, the "industry" of numerical simulations had an impressive development. The first cosmological simulations in the middle 80s used a few $10^{4}$ particles (e.g., 36]). The currently largest simulation (called the Mille- 
nium simulation [11]) uses $\sim 10^{10}$ particles! A main effort is done to reach larger and larger dynamic ranges in order to simulate encompassing volumes large enough to contain representative populations of all kinds of halos (low mass and massive ones, in low- and high-density environments, high-peak rare halos), yet resolving the inner structure of individual halos.

\section{Halo mass function}

The CDM halo mass function (comoving number density of halos of different masses at a given epoch $z, n(M, z))$ obtained in the $\mathrm{N}$-body simulations is consistent with the P-S function in general, which is amazing given the approximate character of the P-S analysis. However, in more detail, the results of large N-body simulations are better fitted by modified P-S analytical functions, as the one derived in 103 and showed in Fig. 8 Using the Millennium simulation, the halo mass function has been accurately measured in the range that is well sampled by this run $\left(z \leq 12, M \geq 1.7 \times 10^{10} \mathrm{M}_{\odot} \mathrm{h}^{-1}\right)$. The mass function is described by a power law at low masses and an exponential cut-off at larger masses. The "cut-off", most typical mass, increases with time and is related to the hierarchical evolution of the $1 \sigma$ halos shown in Fig. 7 The halo mass function is the starting point for modeling the luminosity function of galaxies. From Fig. 8 we see that the evolution of the abundances of massive halos is much more pronounced than the evolution of less massive halos. This is why observational studies of abundance of massive galaxies or cluster of galaxies at high redshifts provide a sharp test to theories of cosmic structure formation. The abundance of massive rare halos at high redshifts are for example a strong function of the fluctuation field primordial statistics (Gaussianity or non-Gaussianity).

Subhalos. An important result of N-body simulations is the existence of subhalos, i.e. halos inside the virial radius of larger halos, which survived as self-bound entities the gravitational collapse of the higher level of the hierarchy. Of course, subhalos suffer strong mass loss due to tidal stripping, but this is probably not relevant for the luminous galaxies formed in the innermost regions of (sub)halos. This is why in the case of subhalos, the maximum circular velocity $V_{m}$ (attained at radii much smaller than the virial radius) is used instead of the virial mass. The $V_{m}$ distribution of subhalos inside clustersized and galaxy-sized halos is similar 83. This distribution agrees with the distribution of galaxies seen in clusters, but for galaxy-sized halos the number of subhalos overwhelms by 1-2 orders of magnitude the observed number of satellite galaxies around galaxies like Milky Way and Andromeda [70, 83.

Fig. 9 (right side) shows the subhalo cumulative $V_{m}$-distribution for a CDM Milky Way-like halo compared to the observed satellite $V_{m}$-distribution. In this Fig. are also shown the $V_{m}$-distributions obtained for the same MilkyWay halo but using the power spectrum of three WDM models with particle masses $m_{X} \approx 0.6,1$, and $1.7 \mathrm{KeV}$. The smaller $m_{X}$, the larger is the freestreaming (filtering) scale, $R_{f}$, and the more substructure is washed out (see 


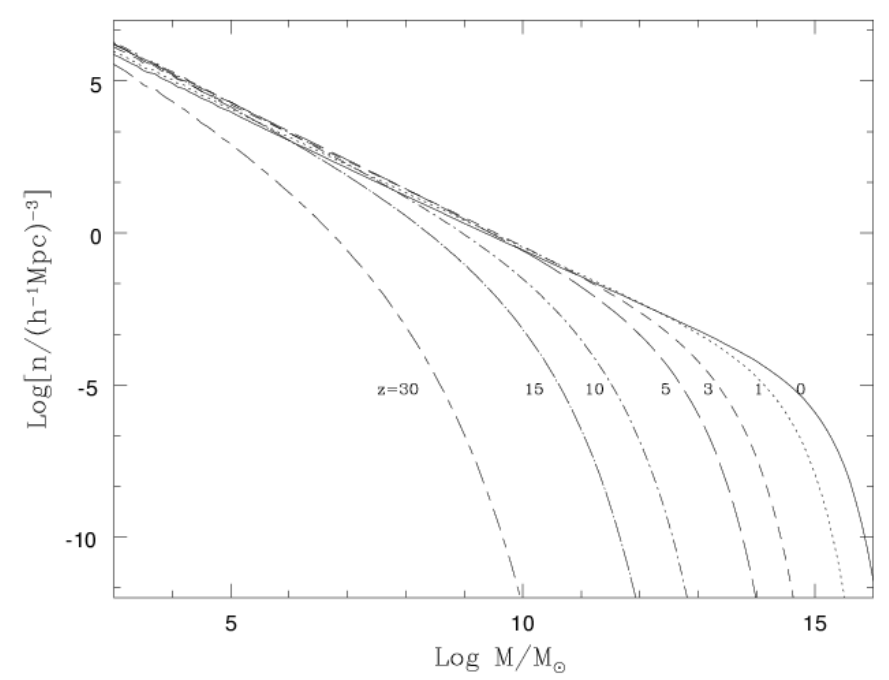

Fig. 8. Evolution of the comoving number density of collapsed halos ( $\mathrm{P}-\mathrm{S}$ mass function) according to the ellipsoidal modification by 103. Note that the "cut-off" mass grows with time. Most of the mass fraction in collapsed halos at a given epoch are contained in halos with masses around the "cut-off" mass.

$\S 3.2)$. In the left side of Fig. [9 is shown the DM distribution inside the MilkyWay halo simulated by using a CDM power spectrum (top) and a WDM power spectrum with $m_{X} \approx 1 \mathrm{KeV}$ (sterile neutrino, bottom). For a student it should be exciting to see with her(his) own eyes this tight connection between micro- and macro-cosmos: the mass of the elemental particle determines the structure and substructure properties of galaxy halos!

\section{Halo density profiles}

High-resolution N-body simulations [87] and semi-analytical techniques (e.g., 3]) allowed to answer the following questions: How is the inner mass distribution in CDM halos? Does this distribution depend on mass? How universal is it? The two-parameter density profile established in [87] (the NavarroFrenk-White, NFW profile) departs from a single power law, and it was proposed to be universal and not depending on mass. In fact the slope $\beta(r) \equiv-\operatorname{dlog} \rho(r) / \operatorname{dlog} r$ of the NFW profile changes from -1 in the center to -3 in the periphery. The two parameters, a normalization factor, $\rho_{s}$ and a shape factor, $r_{s}$, were found to be related in a such a way that the profile depends only on one shape parameter that could be expressed as the concentration, $c_{N F W} \equiv r_{s} / R_{v}$. The more massive the halo, the less concentrated on the average. For the $\Lambda$ CDM model, $c \approx 20-5$ for $M \sim 2 \times 10^{8}-2 \times 10^{15} \mathrm{M}_{\odot} \mathrm{h}^{-1}$, respectively [42. However, for a given $M$, the scatter of $c_{N F W}$ is large $(\approx 30-40 \%)$, and it is related to the halo formation history [3, 21, 125] (see 

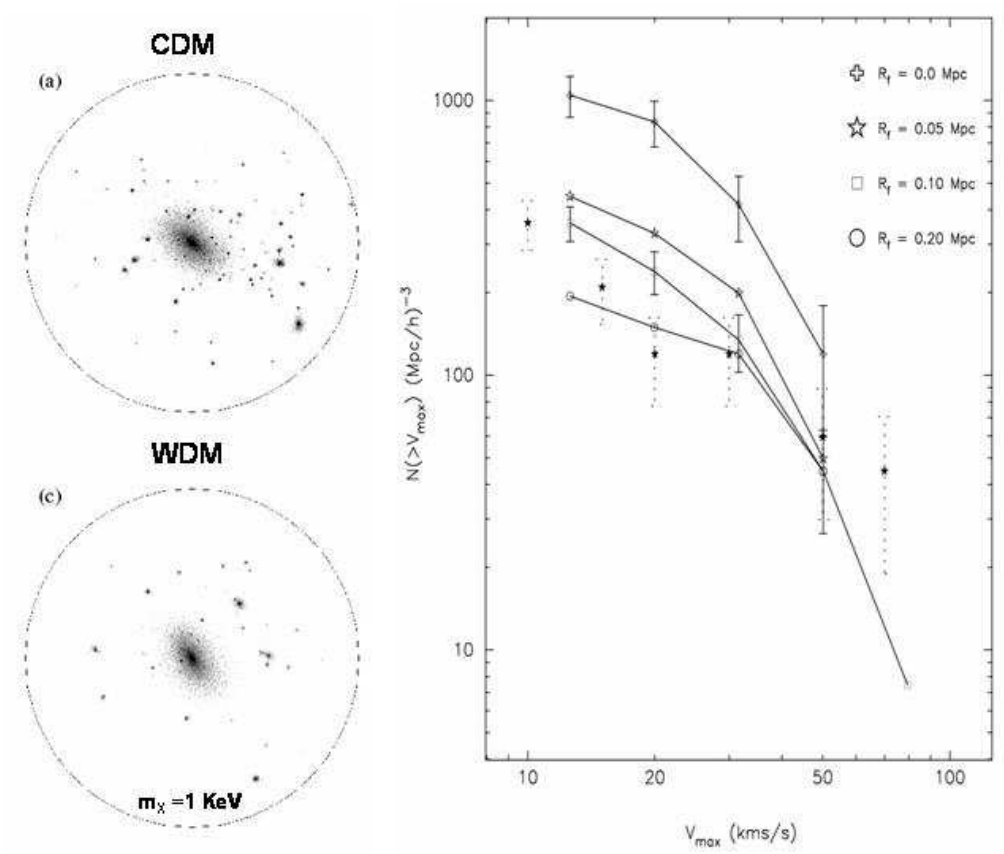

Fig. 9. Dark matter distribution in a sphere of $400 \mathrm{Mpch}^{-1}$ of a simulated Galaxysized halo with CDM (a) and WDM $\left(m_{X}=1 \mathrm{KeV}, \mathrm{b}\right)$. The substructure in the latter case is significantly erased. Right panel shows the cumulative maximum $V_{c}$ distribution for both cases (open crosses and squares, respectively) as well as for an average of observations of satellite galaxies in our Galaxy and in Andromeda (dotted error bars). Adapted from [31].

below). A significant fraction of halos depart from the NFW profile. These are typically not relaxed or disturbed by companions or external tidal forces.

Is there a "cusp" crisis? More recently, it was found that the inner density profile of halos can be steeper than $\beta=-1$ (e.g. 84]). However, it was shown that in the limit of resolution, $\beta$ never is as steep a -1.5 88. The inner structure of CDM halos can be tested in principle with observations of (i) the inner rotation curves of DM dominated galaxies (Irr dwarf and LSB galaxies; the inner velocity dispersion of $\mathrm{dSph}$ galaxies is also being used as a test ), and (ii) strong gravitational lensing and hot gas distribution in the inner regions of clusters of galaxies. Observations suggest that the DM distribution in dwarf and LSB galaxies has a roughly constant density core, in contrast to the cuspy cores of CDM halos (the literature on this subject is extensive; see for recent results [37, 50, 107, 128, and more references therein). If the observational studies confirm that halos have constant-density cores, then either astrophysical mechanisms able to expand the halo cores should work efficiently or the $\Lambda \mathrm{CDM}$ scenario should be modified. In the latter case, one of 
the possibilities is to introduce weakly self-interacting DM particles. For small cross sections, the interaction is effective only in the more dense inner regions of galaxies, where heat inflow may expand the core. However, the gravothermal catastrophe can also be triggered. In 32 it was shown that in order to avoid the gravo-thermal instability and to produce shallow cores with densities approximately constant for all masses, as suggested by observations, the DM cross section per unit of particle mass should be $\sigma_{D M} / m_{X}=0.5-1.0 v_{100}^{-1}$ $\mathrm{cm}^{2} / \mathrm{gr}$, where $v_{100}$ is the relative velocity of the colliding particles in unities of $100 \mathrm{~km} / \mathrm{s} ; v_{100}$ is close to the halo maximum circular velocity, $V_{m}$.

The DM mass distribution was inferred from the rotation curves of dwarf and LSB galaxies under the assumptions of circular motion, halo spherical symmetry, the lack of asymmetrical drift, etc. In recent studies it was discussed that these assumptions work typically in the sense of lowering the observed inner rotation velocity [59, 100, 118. For example, in 118] it is demonstrated that non-circular motions (due to a bar) combined with gas pressure support and projection effects systematically underestimate by up to $50 \%$ the rotation velocity of cold gas in the central $1 \mathrm{kpc}$ region of their simulated dwarf galaxies, creating the illusion of a constant density core.

Mass-velocity relation. In a very simplistic analysis, it is easy to find that $M \propto V_{c}^{3}$ if the average halo density $\rho_{h}$ does not depend on mass. On one hand, $V_{c} \propto(G M / R)^{1 / 2}$, and on the other hand, $\rho_{h} \propto M / R^{3}$, so that $V_{c} \propto$ $M^{1 / 3} \rho_{h}^{1 / 6}$. Therefore, for $\rho_{h}=$ const, $M \propto V_{c}^{3}$. We have seen in $\S 3.2$ that the CDM perturbations at galaxy scales have similar amplitudes (actually $\left.\sigma_{M} \propto \ln M\right)$ due to the stangexpansion effect in the radiation-dominated era. This implies that galaxy-sized perturbations collapse within a small range of epochs attaining more or less similar average densities. The CDM halos actually have a mass distribution that translates into a circular velocity profile $V_{c}(r)$. The maximum of this profile, $V_{m}$, is typically the circular velocity that characterizes a given halo of virial mass $M$. Numerical and semi-numerical results show that $(\Lambda \mathrm{CDM}$ model):

$$
M \approx 5.2 \times 10^{4}\left(\frac{V_{m}}{k m s^{-1}}\right)^{3.2} \mathrm{M}_{\odot} \mathrm{h}^{-1},
$$

Assuming that the disk infrared luminosity $L_{I R} \propto M$, and that the disk maximum rotation velocity $V_{\text {rot }, m} \propto V_{m}$, one obtains that $L_{I R} \propto V_{\text {rot }, m}^{3.2}$, amazingly similar to the observed infrared Tully-Fisher relation [116, one of the most robust and intriguingly correlations in the galaxy world! I conclude that this relation is a clear imprint of the CDM power spectrum of fluctuations.

Mass assembling histories

One of the key concepts of the hierarchical clustering scenario is that cosmic structures form by a process of continuous mass aggregation, opposite to the monolithic collapse scenario. The mass assembly of CDM halos is characterized by the mass aggregation history $(\mathrm{MAH})$, which can alternate smooth 
mass accretion with violent major mergers. The MAH can be calculated by using semi-analytical approaches based on extensions of the P-S formalism. The main idea lies in the estimate of the conditional probability that given a collapsed region of mass $M_{0}$ at $z_{0}$, a region of mass $M_{1}$ embedded within the volume containing $M_{0}$, had collapsed at an earlier epoch $z_{1}$. This probability is calculated based on the excursion set formalism starting from a Gaussian density field characterized by an evolving mass variance $\sigma_{M}$ [17, 173]. By using the conditional probability and random trials at each temporal step, the "backward" MAHs corresponding to a fixed mass $M_{0}$ (defined for instance at $z=0)$ can be traced. The MAHs of isolated halos by definition decrease toward the past, following different tracks (Fig. 10), sometimes with abrupt big jumps that can be identified as major mergers in the halo assembly history.
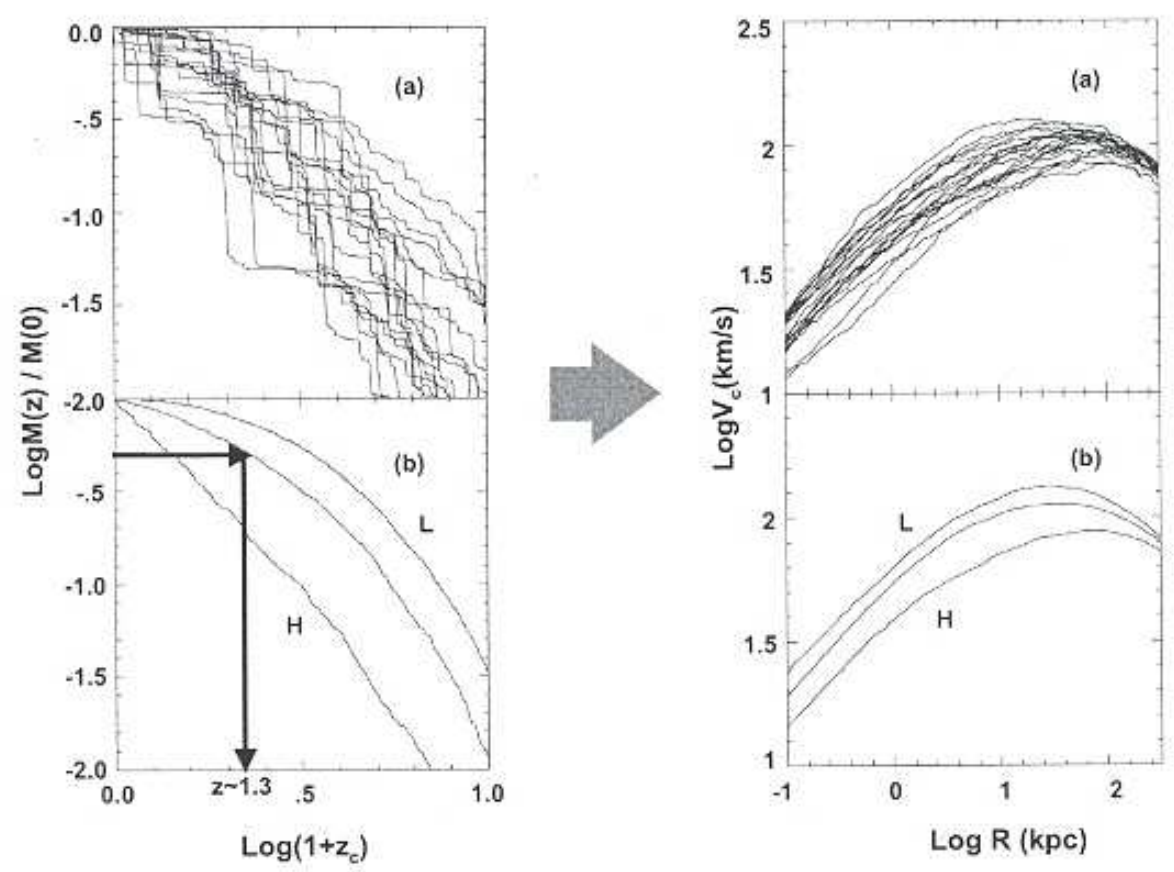

Fig. 10. Upper panels (a). A score of random halo MAHs for a present-day virial mass of $3.5 \times 10^{11} \mathrm{M}_{\odot}$ and the corresponding circular velocity profiles of the virialized halos. Lower panels (b). The average MAH and two extreme deviations from $10^{4}$ random MAHs for the same mass as in (a), and the corresponding halo circular velocity profiles. The MAHs are diverse for a given mass and the $V_{c}$ (mass) distribution of the halos depend on the MAH. Adapted from 45]. 
To characterize typical behaviors of the halo MAHs, one may calculate the average MAH for a given virial mass $M_{0}$, for a given "population" of halos selected by its environment, etc. In the left panels of Fig. 10 are shown 20 individual MAHs randomly selected from $10^{4}$ trials for $M_{0}=3.5 \times 10^{11} \mathrm{M}_{\odot}$ in a $\Lambda \mathrm{CDM}$ cosmology 45. In the bottom panel are plotted the average MAH from these $10^{4}$ trials as well as two extreme deviations from the average. The average MAHs depend on mass: more massive halos have a more extended average MAH, i.e. they aggregate a given fraction of $M_{0}$ latter than less massive halos. It is a convention to define the typical halo formation redshift, $z_{f}$, when half of the current halo mass $M_{0}$ has been aggregated. For instance, for the $\Lambda \mathrm{CDM}$ cosmology the average MAHs show that $z_{f} \approx 2.2,1.2$ and 0.7 for $M_{0}=10^{10} \mathrm{M}_{\odot}, 10^{12} \mathrm{M}_{\odot}$ and $10^{14} \mathrm{M}_{\odot}$, respectively. A more physical definition of halo formation time is when the halo maximum circular velocity $V_{m}$ attains its maximum value. After this epoch, the mass can continue growing, but the inner gravitational potential of the system is already set.

Right panels of Fig. 10 show the present-day halo circular velocity profiles, $V_{c}(r)$, corresponding to the MAHs plotted in the left panels. The average $V_{c}(r)$ is well described by the NFW profile. There is a direct relation between the MAH and the halo structure as described by $V_{c}(r)$ or the concentration parameter. The later the MAH, the more extended is $V_{c}(r)$ and the less concentrated is the halo 3, 125. Using high-resolution simulations some authors have shown that the halo MAH presents two regimes: an early phase of fast mass aggregation (mainly by major mergers) and a late phase of slow aggregation (mainly by smooth mass accretion) [133, 75]. The potential well of a present-day halo is set mainly at the end of the fast, major-merging driven, growth phase.

From the MAHs we may infer: (i) the mass aggregation rate evolution of halos (halo mass aggregated per unit of time at different $z^{\prime}$ s), and (ii) the major merging rates of halos (number of major mergers per unit of time per halo at different $z^{\prime} \mathrm{s}$ ). These quantities should be closely related to the star formation rates of the galaxies formed within the halos as well as to the merging of luminous galaxies and pair galaxy statistics. By using the $\Lambda \mathrm{CDM}$ model, several studies showed that most of the mass of the present-day halos has been aggregated by accretion rather than major mergers (e.g., 85]). Major merging was more frequent in the past [55], and it is important for understanding the formation of massive galaxy spheroids and the phenomena related to this process like QSOs, supermassive black hole growth, obscured star formation bursts, etc. Both the mass aggregation rate and major merging rate histories depend strongly on environment: the denser the environment, the higher is the merging rate in the past. However, in the dense environments (group and clusters) form typically structures more massive than in the less dense regions (field and voids). Once a large structure virializes, the smaller, galaxy-sized halos become subhalos with high velocity dispersions: the mass growth of the subhalos is truncated, or even reversed due to tidal stripping, and the merging probability strongly decreases. Halo assembling (and therefore, galaxy assem- 
bling) definitively depends on environment. Overall, by integrating the MAHs of the whole galaxy-sized $\Lambda \mathrm{CDM}$ halo population in a given volume, the general result is that the peak in halo assembling activity was at $z \approx 1-2$. After these redshifts, the global mass aggregation rate strongly decreases (e.g., 121.

To illustrate the driving role of DM processes in galaxy evolution, I mention briefly here two concrete examples:

1). Distributions of present-day specific mass aggregation rate, $(\dot{M} / M)_{0}$, and halo lookback formation time, $T_{1 / 2}$. For a $\Lambda \mathrm{CDM}$ model, these distributions are bimodal, in particular the former. We have found that roughly $40 \%$ of halos (masses larger than $\approx 10^{11} \mathrm{M}_{\odot} \mathrm{h}^{-1}$ ) have $(\dot{M} / M)_{0} \leq 0$; they are basically subhalos. The remaining $60 \%$ present a broad distribution of $(\dot{M} / M)_{0}>0$ peaked at $\approx 0.04 \mathrm{Gyr}^{-1}$. Moreover, this bimodality strongly changes with large-scale environment: the denser is the environment the, higher is the fraction of halos with $(\dot{M} / M)_{0} \leq 0$. It is interesting enough that similar fractions and dependences on environment are found for the specific star formation rates of galaxies in large statistical surveys $(\S \S 2.3)$; the situation is similar when confronting the distributions of $T_{1 / 2}$ and observed colors. Therefore, it seems that the the main driver of the observed bimodalities in $z=0$ specific star formation rate and color of galaxies is the nature of the CDM halo mass aggregation process. Astrophysical processes of course are important but the main body of the bimodalities can be explained just at the level of DM processes.

2. Major merging rates. The observational inference of galaxy major merging rates is not an easy task. The two commonly used methods are based on the statistics of galaxy pairs (pre-mergers) and in the morphological distortions of ellipticals (post-mergers). The results show that the merging rate increases as $(1+z)^{x}$, with $x \sim 0-4$. The predicted major merging rates in the $\Lambda \mathrm{CDM}$ scenario agree roughly with those inferred from statistics of galaxy pairs. From the fraction of normal galaxies in close companions (with separations less than $50 \mathrm{kpch}^{-1}$ ) inferred from observations at $z=0$ and $z=0.3$ 91, and assuming an average merging time of $\sim 1$ Gyr for these separations, we estimate that the major merging rate at the present epoch is $\sim 0.01 \mathrm{Gyr}^{-1}$ for halos in the range of $0.1-2.010^{12} \mathrm{M}_{\odot}$, while at $z=0.3$ the rate increased to $\sim 0.018 \mathrm{Gyr}^{-1}$. These values are only slightly lower than predictions for the $\Lambda \mathrm{CDM}$ model.

\section{Angular momentum}

The origin of the angular momentum (AM) is a key ingredient in theories of galaxy formation. Two mechanisms of AM acquirement were proposed for the CDM halos (e.g., 93, 22, 78 ): 1. tidal torques of the surrounding shear field when the perturbation is still in the linear regime, and 2. transfer of orbital AM to internal AM in major and minor mergers of collapsed halos. The angular momentum of DM halos is parametrized in terms of the dimensionless spin parameter $\lambda \equiv J \sqrt{E} /\left(G M^{5 / 2}\right.$, where $J$ is the modulus of the total angular 
momentum and $E$ is the total (kinetic plus potential). It is easy to show that $\lambda$ can be interpreted as the level of rotational support of a gravitational system, $\lambda=\omega / \omega_{\text {sup }}$, where $\omega$ is the angular velocity of the system and $\omega_{\text {sup }}$ is the angular velocity needed for the system to be rotationally supported against gravity (see 90]).

For disk and elliptical galaxies, $\lambda \sim 0.4-0.8$ and $\sim 0.01-0.05$, respectively. Cosmological N-body simulations showed that the CDM halo spin parameter is $\log$-normal distributed, with a median value $\lambda \approx 0.04$ and a standard deviation $\sigma_{\lambda} \approx 0.5$; this distribution is almost independent from cosmology. A related quantity, but more straightforward to compute is $\lambda^{\prime} \equiv \frac{J}{\sqrt{2} M V_{v} R_{v}}$ [22], where $R_{v}$ is the virial radius and $V_{v}$ the circular velocity at this radius. Recent simulations show that $\left(\lambda^{\prime}, \sigma_{\lambda^{\prime}}\right) \approx(0.035,0.6)$, though some variations with environment and mass are measured [5]. The evolution of the spin parameter depends on the AM acquirement mechanism. In general, a significant systematical change of $\lambda$ with time is not expected, but relatively strong changes are measured in short time steps, mainly after merging of halos, when $\lambda$ increases.

How is the internal AM distribution in CDM halos? Bullock et al. 22. found that in most of cases this distribution can be described by a simple (universal) two-parameter function that departs significantly from the solidbody rotation distribution. In addition, the spatial distribution of AM in CDM halos tends to be cylindrical, being well aligned for $80 \%$ of the halos, and misaligned at different levels for the rest. The mass distribution of the galaxies formed within CDM halos, under the assumption of specific AM conservation, is established by $\lambda$, the halo AM distribution, and its alignment.

\subsection{Non-baryonic dark matter candidates}

The non-baryonic DM required in cosmology to explain observations and cosmic structure formation should be in form of elemental or scalar field particles or early formed quark nuggets. Modifications to fundamental physical theories (modified Newtonian Dynamics, extra-dimensions, etc.) are also plausible if DM is not discovered.

There are several docens of predicted elemental particles as DM candidates. The list is reduced if we focus only on well-motivated exotic particles from the point of view of particle physics theory alone (see for a recent review [53]). The most popular particles beyond the standard model are the supersymmetric (SUSY) particles in supersymmetric extensions of the Standard Model of particle physics. Supersymmetry is a new symmetry of space-time introduced in the process of unifying the fundamental forces of nature (including gravity). An excellent CDM candidate is the lightest stable SUSY particle under the requirement that superpartners are only produced or destroyed in pairs (called R-parity conservation). This particle called neutralino is weakly interacting and massive (WIMP). Other SUSY particles are the gravitino and the sneutrino; they are of WDM type. The predicted masses for neutralino range from $\sim 30$ to $5000 \mathrm{GeV}$. The cosmological density of neutralino (and of 
other thermal WIMPs) is naturally as required when their interaction cross section is of the order of a weak cross section. The latter gives the possibility to detect neutralinos in laboratory.

The possible discovery of WIMPs relies on two main techniques:

(i) Direct detections. The WIMP interactions with nuclei (elastic scattering) in ultra-low-background terrestrial targets may deposit a tiny amount of energy $(<50 \mathrm{keV})$ in the target material; this kinetic energy of the recoiling nucleus is converted partly into scintillation light or ionization energy and partly into thermal energy. Dozens of experiments worldwide -of cryogenic or scintillator type, placed in mines or underground laboratories, attempt to measure these energies. Predicted event rates for neutralinos range from $10^{-6}$ to 10 events per kilogram detector material and day. The nuclear recoil spectrum is featureless, but depends on the WIMP and target nucleus mass. To convincingly detect a WIMP signal, a specific signature from the galactic halo particles is important. The Earth's motion through the galaxy induces both a seasonal variation of the total event rate and a forward-backward asymmetry in a directional signal. The detection of structures in the dark velocity space, as those predicted to be produced by the Sagittarius stream, is also an specific signature from the Galactic halo; directional detectors are needed to measure this kind of signatures.

The DAMA collaboration reported a possible detection of WIMP particles obeying the seasonal variation; the most probable value of the WIMP mass was $\sim 60 \mathrm{GeV}$. However, the interpretation of the detected signal as WIMP particles is controversial. The sensitivity of current experiments (e.g., CDMS and EDEL-WEISS) limit already the WIMP-proton spin-independent cross

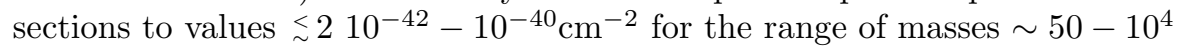
$\mathrm{GeV}$, respectively; for smaller masses, the cross-section sensitivities are larger, and WIMP signals were not detected. Future experiments will be able to test the regions in the cross-section-WIMP mass diagram, where most of models make certain predictions.

(ii) Indirect detections. We can search for WIMPS by looking for the products of their annihilation. The flux of annihilation products is proportional to the square of the WIMP density, thus regions of interest are those where the WIMP concentration is relatively high. There are three types of searches according to the place where WIMP annihilation occur: (i) in the Sun or the Earth, which gives rise to a signal in high-energy neutrinos; (ii) in the galactic halo, or in the halo of external galaxies, which generates $\gamma$-rays and other cosmic rays such as positrons and antiprotons; (iii) around black holes, specially around the black hole at the Galactic Center. The predicted radiation fluxes depend on the particle physics model used to predict the WIMP candidate and on astrophysical quantities such as the dark matter halo structure, the presence of sub-structure, and the galactic cosmic ray diffusion model.

Most of WIMPS were in thermal equilibrium in the early Universe (thermal relics). Particles which were produced by a non-thermal mechanism and that 
never had the chance of reaching thermal equilibrium are called non-thermal relics (e.g., axions, solitons produced in phase transitions, WIMPZILLAs produced gravitationally at the end of inflation). From the side of WDM, the most popular candidate are the $\sim 1 \mathrm{KeV}$ sterile neutrinos. A sterile neutrino is a fermion that has no standard model interactions other than a coupling to the standard neutrinos through their mass generation mechanism. Cosmological probes, mainly the power spectrum of Ly $\alpha$ forest at high redshifts, constrain the mass of the sterile neutrino to values larger than $\sim 2 \mathrm{KeV}$.

\section{The bright side of galaxy formation and evolution}

The $\Lambda \mathrm{CDM}$ scenario of cosmic structure formation has been well tested for perturbations that are still in the linear or quasilinear phase of evolution. These tests are based, among other cosmological probes, on accurate measurements of:

- the CMBR temperature fluctuations at large and small angular scales

- the large-scale mass power spectrum as traced by the spatial distribution of galaxies and cluster of galaxies, by the Ly $\alpha$ forest clouds, by maps of gravitational weak and strong lensing, etc.

- the peculiar large-scale motions of galaxies ${ }^{13}$.

- the statistics of strong gravitational lensing (multiple-lensed arcs).

Although these cosmological probes are based on observations of luminous (baryonic) objects, the physics of baryons plays a minor or indirect role in the properties of the linear mass perturbations. The situation is different at small (galaxy) scales, where perturbations went into the non-linear regime and the dissipative physics of baryons becomes relevant. The interplay of DM and baryonic processes is crucial for understanding galaxy formation and evolution. The progress in this field was mostly heuristic; the $\Lambda \mathrm{CDM}$ scenario provides the initial and boundary conditions for modeling galaxy evolution, but the complex physics of the baryonic processes, in the absence of fundamental theories, requires a model adjustment through confrontation with the observations.

Following, I will outline some key concepts, ingredients, and results of the galaxy evolution study based on the $\Lambda \mathrm{CDM}$ scenario. Some of the pioneer papers in this field are those of Gunn [57, White \& Reese [131], Fall \& Efstathiou [43, Blumental et al. [15], Davis et al. [36, Katz \& Gunn [65], White \& Frenk [130, Kauffmann et al. 66. For useful lecture notes and recent reviews see e.g., Longair 76, 77], White [129], Steinmetz [13], Firmani \& Avila-Reese [46.

$\overline{13}$ Recall that linear theory relates the peculiar velocity, that is the velocity deviation from the Hubble flow, to the density contrast. It is said that the cosmological velocity field is potential; any primordial rotational motion able to give rise to a density perturbation decays as the Universe expands due to angular momentum conservation. 
The main methods of studying galaxy formation and evolution in the $\Lambda \mathrm{CDM}$ context are:

- Semi-analytical Models (e.g., 130, 66, 28, 9, 108, 29, 12, 10]), where the halo mass assembling histories are calculated with the extended PressSchechter formalism and galaxies are seeded within the halos by means of phenomenological recipes. This method is very useful for producing whole populations of galaxies at a given epoch and predicting statistical properties as the luminosity function and the morphological mix.

- Semi-numerical Models (e.g, 45, 2, 119, 16]), where the internal physics of the galaxies, including those of the halos, are modeled numerically but under simplifying assumptions; the initial and boundary conditions are taken from the $\Lambda \mathrm{CDM}$ scenario by using the extended Press-Schechter formalism and halo AM distributions from simulations. This method is useful to predict the local properties of galaxies and correlations among the global properties, as well as to follow the overall evolution of individual galaxies.

- Numerical N-body+hydrodyamical simulations (e.g., 65, 26, 64, 86, 112 126, 1, 110, 56 ), where the DM and baryonic processes are followed in cosmological simulations. This is the most advanced and complete approach to galaxy evolution. However, current limitations in the computational capabilities and the lack of fundamental theories for several of the physical processes involved, do not allow yet to exploit optimally this method. A great advance is being made currently with an hybrid approach: in the high-resolution cosmological N-body simulations of only DM, galaxies are grafted by using the semi-analytical models (e.g., 67, 60, 38, 13, 111, 63]).

\subsection{Disks}

The formation of galaxy disks deep inside the CDM halos is a generic process in the $\Lambda \mathrm{CDM}$ scenario. Let us outline the (simplified) steps of disk galaxy formation in this scenario:

1. DM halo growth. The "mold" for disk formation is provided by the mass and AM distributions of the virialized halo, which grows hierarchically. A description of these aspects were presented in the previous Section.

2. Gas cooling and infall, and the maximum mass of galaxies. It is common to assume that the gas in a halo is shock-heated during collapse to the virial temperature [131]. The gas then cools radiatively and falls in a free-fall time to the center. The cooling function $\Lambda\left(n, T_{k} ; Z\right)$ depends on the gas density, temperature, and composition ${ }^{14}$. Since the seminal work by White \& Frenk (1990) [130, the rate infall of gas available to form the galaxy is assumed to

$\overline{14}$ The main cooling processes for the intrahalo gas are collisional excitation and ionization, recombination, and bremsstrahlung. The former is the most efficient for kinetic temperatures $T_{k} \approx 10^{4}-10^{5} \mathrm{~K}$ and for neutral hydrogen and single ionized helium; for a meta-enriched gas, cooling is efficient at temperatures between $10^{5}-10^{7} \mathrm{~K}$. At higher temperatures, where the gas is completely ionized, the 
be driven either by the free-fall time, $t_{f f}$, if $t_{f f}>t_{\text {cool }}$ or by the cooling time $t_{\text {cool }}$ if $t_{f f}<t_{\text {cool }}$. The former case applies to halos of masses smaller than approximately $5 \times 10^{11} \mathrm{M}_{\odot}$, whilst the latter applies to more massive halos. The cooling flow from the quasistatic hot atmosphere is the process that basically limits the baryonic mass of galaxies [105, and therefore the bright end of the galaxy luminosity function; for the outer, dilute hot gas in large halos, $t_{\text {cool }}$ becomes larger than the Hubble time. However, detailed calculations show that even so, in massive halos too much gas cools, and the bright end of the predicted luminosity function results with a decrease slower than the observed one 12. Below we will see some solutions proposed to this problem.

More recently it was shown that the cooling of gas trapped in filaments during the halo collapse may be so rapid that the gas flows along the filaments to the center, thus avoiding shock heating 69 . However, this process is efficient only for halos less massive than $2.5 \times 10^{11} \mathrm{M}_{\odot}$, which in any case (even if shock-heating happens), cool their gas very rapidly [19]. Thus, for modeling the formation of disks, and for masses smaller than $\sim 5 \times 10^{11} \mathrm{M}_{\odot}$, we may assume that gas infalls in a dynamical time since the halo has virialized, or in two dynamical times since the protostructure was at its maximum expansion.

3. Disk formation, the origin of exponentially, and rotation curves. The gas, originally distributed in mass and AM as the DM, cools and collapses until it reaches centrifugal balance in a disk. Therefore, assuming detailed AM conservation, the radial mass distribution of the disk can be calculated by equating its specific $\mathrm{AM}$ to the $\mathrm{AM}$ of its final circular orbit in centrifugal equilibrium. The typical collapse factor of the gas within a DM halo is $10-15^{15}$, depending on the initial halo spin parameter $\lambda$; the higher the $\lambda$, the more extended (lower surface density) is the resulting disk. The surface density profile of the disks formed within CDM halos is nearly exponential, which provides an explanation to the long-standing question of why galaxy disks are exponential. This is a direct consequence of the AM distribution acquired by the halos by tidal torques and mergers. In more detail, however, the profiles are more concentrated in the center and with a slight excess in the periphery than the exponential law [45, 22. The cusp in the central disk could give rise to either a photometrical bulge [120] or to a real kinematical bulge due to disk gravitational instability enhanced by the higher central surface density [2] (bulge secular formation). In a few cases (high- $\lambda$, low-concentrated halos), purely exponential disks can be formed.

Baryons are a small mass fraction in the CDM halos, however, the disk formed in the center is very dense (recall the high collapse factors), so that

dominant cooling process is bremsstrahlung. At temperatures lower than $10^{4} \mathrm{~K}$ (small halos) and in absence of metals, the main cooling process is by $\mathrm{H}_{2}$ and $H D$ molecule line emission.

${ }^{15}$ It is interesting to note that in the absence of a massive halo around galaxies, the collapse factor would be larger by $\sim M / M_{d} \approx 20$, where $M$ and $M_{d}$ are the total halo and disk masses, respectively 90 . 
the contribution of the baryonic disk to the inner gravitational potential is important or even dominant. The formed disk will drag gravitationally DM, producing an inner halo contraction that is important to calculate for obtaining the rotation curve decomposition. The method commonly used to calculate it is based on the approximation of radial adiabatic invariance, where spherical symmetry and circular orbits are assumed (e.g., 47, 82]). However, the orbits in CDM halos obtained in N-body simulations are elliptical rather than circular; by generalizing the adiabatic invariance to elliptical orbits, the halo contraction becomes less efficient [132, 52].

The rotation curve decomposition of disks within contracted $\Lambda \mathrm{CDM}$ halos are in general consistent with observations 82, 45, 132] (nearly-flat total rotation curves; maximum disk for high-surface brightness disks; submaximum disk for the LSB disks; in more detail, the outer rotation curve shape depends on surface density, going from decreasing to increasing at the disk radius for higher to lower densities, respectively). However, there are important nonsolved issues. For example, from a large sample of observed rotation curves, Persic et al. 95] inferred that the rotation curve shapes are described by an "universal" profile that (i) depends on the galaxy luminosity and (ii) implies a halo profile different from the CDM (NFW) profile. Other studies confirm only partially these claims $123,132,25$. Statistical studies of rotation curves are very important for testing the $\Lambda \mathrm{CDM}$ scenario.

In general, the structure and dynamics of disks formed within $\Lambda \mathrm{CDM}$ halos under the assumption of detailed AM conservation seem to be consistent with observations. An important result to remark is the successful prediction of the infrared Tully-Fisher relation and its scatter ${ }^{16}$. The core problem mentioned in $\S 4.2$ is the most serious potential difficulty. Other potential difficulties are: (i) the predicted disk size (surface brightness) distribution implies a $P(\lambda)$ distribution narrower than that corresponding to $\Lambda \mathrm{CDM}$ halos by almost a factor of two [74; (ii) the internal AM distribution inferred from observations of dwarf galaxies seems not to be in agreement with the $\Lambda \mathrm{CDM}$ halo $\mathrm{AM}$ distribution [122]; (iii) the inference of the halo profile from the statistical study of rotation curve shapes seems not to be agreement with CMD halos. In $\mathrm{N}$-body+hydrodynamical simulations of disk galaxy formation there was common another difficulty called the 'angular momentum catastrophe': the simulated disks ended too much concentrated, apparently due to AM transference of baryons to DM during the gas collapse. The formation of highly concentrated disks also affects the shape of the rotation curve (strongly decreasing), as well as the zero-point of the Tully-Fisher relation. Recent nu-

\footnotetext{
${ }^{16}$ In $\S 4.1$ we have shown that the basis of the Tully-Fisher relation is the CDM halo $M-V_{m}$ relation. From the pure halo to the disk+halo system there are several intermediate processes that could distort the original $M-V_{m}$ relation. However, it was shown that the way in which the CDM halo couples with the disk and the way galaxies transform their gas into stars "conspire" to keep the relation. Due to this conspiring, the Tully-Fisher relation is robust to variations in the baryon fraction $f_{B}$ (or mass-to-luminosity ratios) and in the spin parameter $\lambda$ [4].
} 
merical simulations are showing that the 'angular momentum catastrophe', rather than a physical problem, is a problem related to the resolution of the simulations and the correct inclusion of feedback effects.

4. Star formation and feedback. We are coming to the less understood and most complicated aspects of the models of galaxy evolution, which deserve separate notes. The star formation (SF) process is studied at two levels (each one by two separated communities!): (i) the small-scale physics, related to the complex processes by which the cold gas inside molecular clouds fragments and collapses into stars, and (ii) the large-scale physics, related to the disk global instabilities that give rise to the largest unities of SF, the molecular clouds. The SF physics incorporated to galaxy evolution models is still oversimplified, phenomenological and refers to the latter item. The large-scale SF cycle in normal galaxies is believed to be self-regulated by a balance between the energy injection due to SF (mainly $\mathrm{SNe}$ ) and dissipation (radiative or turbulent). Two main approaches have been used to describe the SF self-regulation in models of galaxy evolution: (a) the halo cooling-feedback approach [130]), (b) the disk turbulent ISM approach [44, 124.

According to the former, the cool gas is reheated by the "galaxy" SF feedback and driven back to the intrahalo medium until it again cools radiatively and collapses into the galaxy. This approach has been used in semi-analytical models of galaxy formation where the internal structure and hydrodynamics of the disks are not treated in detail. The reheating rate is assumed to depend on the halo circular velocity $V_{c}: \dot{M}_{r h} \propto \dot{M}_{s} / V_{c}^{\alpha}$, where $\dot{M}_{s}$ is the SF rate (SFR) and $\alpha \geq 2$. Thus, the galaxy SFR, gas fraction and luminosity depend on $V_{c}$. In these models, the disk ISM is virtually ignored and the SN-energy injection is assumed to be as efficient as to reheat the cold gas up to the virial temperature of the halo. A drawback of the model is that it predicts hot X-ray halos around disk galaxies much more luminous than those observed.

Approach (b) is more appropriate for models where the internal processes of the disk are considered. In this approach, the SF at a given radius $r$ is assumed to be triggered by disk gravitational instabilities (Toomre criterion) and self-regulated by a balance between energy injection (mainly by $\mathrm{SNe}$ ) and dissipation in the turbulent ISM in the direction perpendicular to the disk plane:

$$
\begin{array}{r}
Q_{g}(r) \equiv \frac{v_{g}(r) \kappa(r)}{\pi G \Sigma_{g}(r)}<Q_{c r i t} \\
\gamma_{S N} \epsilon_{S N} \dot{\Sigma}_{*}(r)+\dot{\Sigma}_{E, a c c r}(r)=\frac{\Sigma_{g}(r) v_{g}^{2}(r)}{2 t_{d}(r)},
\end{array}
$$

where $v_{g}$ and $\Sigma_{g}$ are the gas velocity dispersion and surface density, $\kappa$ is the epicyclic frequency, $Q_{c r i t}$ is a critical value for instability, $\gamma_{S N}$ and $\epsilon_{S N}$ are the kinetic energy injection efficiency of the SN into the gas and the SN energy generated per gram of gas transformed into stars, respectively, $\dot{\Sigma}_{*}$ is the surface SFR, and $\dot{\Sigma}_{E, a c c r}$ is the kinetic energy input due to mass 
accretion rate (or eventually any other energy source as AGN feedback). The key parameter in the self-regulating process is the dissipation time $t_{d}$. The disk ISM is a turbulent, non-isothermal, multi-temperature flow. Turbulent dissipation in the ISM is typically efficient $\left(t_{d} \sim 10^{7}-10^{8} \mathrm{yr}\right)$ in such a way that self-regulation happens at the characteristic vertical scales of the disk. Thus, there is not too much room for strong feedback with the gas at heights larger than the vertical scaleheigth of normal present-day disks: self-regulation is at the level of the disk, but not at the level of the gas corona around. With this approach the predicted SFR is proportional to $\Sigma_{g}^{n}$ (Schmidt law), with $n \approx 1.4-2$ varying along the disk, in good agreement with observational inferences. The typical SF timescales are not longer than 3-4Gyr. Therefore, to keep active SFRs in the disks, gas infall is necessary, a condition perfectly fulfilled in the $\Lambda \mathrm{CDM}$ scenario.

Given the SFR radius by radius and time by time, and assuming an IMF, the corresponding luminosities in different color bands can be calculated with stellar population synthesis models. The final result is then an evolving insideout luminous disk with defined global and local colors.

\section{Secular evolution}

The "quiet" evolution of galaxy disks as described above can be disturbed by minor mergers (satellite accretion) and interactions with close galaxy companions. However, as several studies have shown, the disk may suffer even intrinsic instabilities which lead to secular changes in its structure, dynamics, and SFR. The main effects of secular evolution, i.e. dynamical processes that act in a timescale longer than the disk dynamical time, are the vertical thickening and "heating" of the disk, the formation of bars, which are efficient mechanisms of radial AM and mass redistribution, and the possible formation of (pseudo)bulges (see for recent reviews [71, 33]). Models of disk galaxy evolution should include these processes, which also can affect disk properties, for example increasing the disk scale radii [117].

\subsection{Spheroids}

As mentioned in $\S 2$, the simple appearance, the dominant old stellar populations, the $\alpha$-elements enhancement, and the dynamically hot structure of spheroids suggest that they were formed by an early $(z>4)$ single violent event with a strong burst of star formation, followed by passive evolution of their stellar population (monolithic mechanism). Nevertheless, both observations and theory point out to a more complex situation. There are two ways to define the formation epoch of a spheroid: when most of its stars formed or when the stellar spheroid acquired its dynamical properties in violent or secular processes. For the monolithic collapse mechanism both epochs coincide.

In the context of the $\Lambda \mathrm{CDM}$ scenario, spheroids are expected to be formed basically as the result of major mergers of disks. However, 
- if the major mergers occur at high redshifts, when the disks are mostly gaseous, then the situation is close to the monolithic collapse;

- if the major mergers occur at low redshifts, when the galaxies have already transformed a large fraction of their gas into stars, then the spheroids assemble by the "classical" dissipationless collision.

Besides, stellar disks may develop spheroids in their centers (bulges) by secular evolution mechanisms, both intrinsic or enhanced by minor mergers and interactions; this channel of spheroid formation should work for latetype galaxies and it is supported by a large body of observations [71]. But the picture is even more complex in the hierarchical cosmogony as galaxy morphology may be continuously changing, depending on the MAH (smooth accretion and violent mergers) and environment. An spheroid formed early should continue accreting gas so that a new, younger disk grows around. A naive expectation in the context of the $\Lambda \mathrm{CDM}$ scenario is that massive elliptical galaxies should be assembled mainly by late major mergers of the smaller galaxies in the hierarchy. It is also expected that the disks in galaxies with small bulge-to-disk ratios should be on average redder than those in galaxies with large bulge-to-disk ratios, contrary to observations.

Although it is currently subject of debate, a more elaborate picture of spheroid formation is emerging now in the context of the $\Lambda \mathrm{CDM}$ hierarchical scenario (see 106, 46, 39] and the references therein). The basic ideas are that massive ellipticals formed early $(z>3)$ and in a short timescale by the merging of gas-rich disks in rare high-peak, clustered regions of the Universe. The complex physics of the merging implies (i) an ultraluminous burst of SF obscured by dust (cool ULIRG phase) and the establishment of a spheroidal structure, (ii) gas collapse to the center, a situation that favors the growth of the preexisting massive black hole(s) through an Eddington or even superEddington regime (warm ULIRG phase), (iii) the switch on of the AGN activity associated to the supermassive black hole when reaching a critical mass, reverting then the gas inflow to gas outflow (QSO phase), (iv) the switch off of the AGN activity leaving a giant stellar spheroid with a supermassive black hole in the center and a hot gas corona around (passive elliptical evolution). In principle, the hot corona may cool by cooling flows and increase the mass of the galaxy, likely renewing a disk around the spheroid. However, it seems that recurrent AGN phases (less energetic than the initial QSO phase) are possible during the life of the spheroid. Therefore, the energy injected from AGN in the form of radio jets (feedback) can be responsible for avoiding the cooling flow. This way is solved the problem of disk formation around the elliptical, as well as the problem of the extended bright end in the luminosity function. It is also important to note that as soon as the halo hosting the elliptical becomes a subhalo of the group or cluster, the MAH is truncated $(\S 4)$. According to the model just described, massive elliptical galaxies were in place at high redshifts, while less massive galaxies (collapsing from more common density peaks) assembled later. This model was called downsizing or 
anti-hierarchical. In spite of the name, it fits perfectly within the hierarchical $\Lambda \mathrm{CDM}$ scenario.

\subsection{Drivers of the Hubble sequence}

- Disks are generic objects formed by gas dissipation and collapse inside the growing CDM halos. Three (cosmological) initial and boundary conditions related to the halos define the main properties of disks in isolated halos:

1. The virial mass, which determines extensive properties

2 . The spin parameter $\lambda$, which determines mainly the disk surface brightness (SB; it gives rise to the sequence from high SB to low SB disks) and strongly influences the rotation curve shape and the bulgeto-disk ratio (within the secular scenario). $\lambda$ also plays some role in the SFR history.

3. The MAH, which drives the gas infall rate and, therefore, the disk SFR and color; the MAH determines also the halo concentration, and its scatter is reflected in the scatter of the Tully-Fisher relation.

The two latter determine the intensive properties of disks, suggesting a biparametrical sequence in SB and color. There is a fourth important parameter, the galaxy baryon fraction $f_{B}$, which influences the disk SB and rotation curve shape. We have seen that $f_{B}$ in galaxies is 3-5 times lower than the universal $\Omega_{B} / \Omega_{D M}$ fraction. This parameter is related probably to astrophysical processes as gas dissipation and feedback.

- The clustering of CDM halos follows an spatial distribution with very different large-scale environments. In low-density environments, halos live mostly isolated, favoring the formation of disks, whose properties are driven by the factors mentioned above. However, as we move to higher-density environments, halos form from more and more clustered high-peak perturbations that assemble early by violent major mergers: this is the necessary condition to form massive ellipticals. At some time, the larger scale in the hierarchy collapses and the halo becomes a subhalo: the mass aggregation is then truncated and the probability of merging decreases dramatically. Elliptical galaxies are settled and continue evolving passively. Thus, the environment of CDM halos is another important driver of the Hubble sequence, able to establish the main body of the observed blue-red and early-type morphology sequences and their dependences on density.

- Although the initial, boundary and environmental conditions provided by the $\Lambda \mathrm{CDM}$ scenario are drivers of several of the main properties and correlations of galaxies, astrophysical processes should also play an important role. The driving astrophysical processes are global SF and feedback. They should come in two modes that drive the disk and elliptical sequences: (i) the quiescent disk mode, where disk instabilities trigger SF and local (negative) feedback self-regulates the SFR, and (ii) the bursting mode of violent mergers of gaseous galaxies, where local shocks and gravothermal catastrophe trigger SF, and presumably a positive feedback increases its efficiency. Other 
important astrophysical drivers of galaxy properties are: (i) the SN-induced wind-driven outflows, which are important to shape the properties of dwarf galaxies $\left(M \lesssim 10^{10} \mathrm{M}_{\odot}, V_{m} \lesssim 80 \mathrm{~km} / \mathrm{s}\right)$, (ii) the AGN-induced hydrodynamical outflows, which are important to prevent cooling flows in massive ellipticals, (iii) several processes typical of high-density environments such as ram pressure, harassment, strangulation, etc., presumably important to shape some properties of galaxies in clusters.

\section{Issues and outlook}

Our understanding of galaxy formation and evolution is in its infancy. So far, only the first steps were given in the direction of consolidating a theory in this field. The process is apparently so complex and non-linear that several specialists do not expect the emergence of a theory in the sense that a few driving parameters and factors might explain the main body of observations. Instead, the most popular trend now is to attain some description of galaxy evolution by simulating it in expensive computational runs. I believe that simulations are a valuable tool to extend a bridge between reality and the distorted (biased) information given by observations. However, the search of basic theories for explaining galaxy formation and evolution should not be replaced by the only effort of simulating in detail what in fact we want to get. The power of science lies in its predictive capability. Besides, if galaxy theory becomes predictive, then its potential to test fundamental and cosmological theories will be enormous.

Along this notes, potential difficulties or unsolved problems of the $\Lambda \mathrm{CDM}$ scenario were discussed. Now I summarize and complement them:

\section{Physics}

- What is non-baryonic DM? From the structure formation side, the preferred (and necessary!) type is CDM, though WDM with filtering masses below $10^{9} \mathrm{M}_{\odot}$ is also acceptable. So far none of the well-motivated cold or warm nonbaryonic particles have been detected in Earth experiments. The situation is even worth for proposals not based on elemental particles as DM from extradimensions.

- What is Dark Energy? Dark Energy does not play apparently a significant role in the internal evolution of perturbations but it crucially defines the cosmic timescale and expansion rate, which are important for the growing factor of perturbations. The simplest interpretation of Dark Energy is the homogeneous and inert cosmological constant $\Lambda$, with equation of state parameter $w=-1$ and $\rho_{\Lambda}=$ const. The combinations of different cosmological probes tend to favor the flat-geometry $\Lambda$ models with $\left(\Omega_{M}, \Omega_{\Lambda}\right) \approx(0.26,0.74)$. However, the cosmological constant explanation of Dark Energy faces serious theoretical problems. Several alternatives to $\Lambda$ were proposed to ameliorate 
partially these problems (e.g. quintaessence, k-essence, Chaplygin gas, etc.). Also have been proposed unifying schemes of DM and Dark Energy through scalar fields (e.g, 81]).

\section{Cosmology}

- Inflation provides a natural mechanism for the generation of primordial fluctuations. The nearly scale-invariance of the primordial power spectrum is well predicted by several inflation models, but its amplitude, rather than being predicted, is empirically inferred from observations of CMBR anisotropies. Another aspect of primordial fluctuations not well understood is related to their statistics, i.e., whether they are Gaussian-distributed or not. And this is crucial for cosmic structure formation.

- Indirect pieces of evidence are consistent with the main predictions of inflation regarding primordial fluctuations. However, more direct tests of this theory are highly desirable. Hopefully, CMBR anisotropy observations will allow for some more direct tests (e.g., effects from primordial gravitational waves).

\section{Astrophysics}

- Issues at small scales. The excess of substructure (satellite galaxies) can be apparently solved by inhibition of galaxy formation in small halos due to UV-radiation produced by reionization and due to feedback, rather than to modifications to the scenario (e.g., the introduction of WDM). Observational inferences of the inner volume and phase-space densities of dwarf satellite galaxies are crucial to explore this question. The direct detection (with gravitational lensing) of the numerous subhalo (dark galaxy) population predicted by CDM for the Galaxy halo is a decisive test on the problem of substructure. The CDM prediction of cuspy halos is a more involved problem when confronting it with observational inferences. If the disagreement persists, then either the $\Lambda \mathrm{CDM}$ scenario will need a modification (e.g., introduction of selfinteraction or annihilation), or astrophysical processes involving gas baryon physics should be in action. However, there are still unsolved issues at the intermediate level: for example, the central halo density profile of galaxies is inferred from observations of inner rotation curves under several assumptions that could be incorrect. An interesting technique to overcome this problem is being currently developed: to simulate as realistically as possible a given galaxy, "observe" its rotation curve and then compare with that of the real galaxy (see $\S \S 4.1)$.

- The early formation of massive red elliptical galaxies can be accommodated in the hierarchical $\Lambda \mathrm{CDM}$ scenario $(\S \S 5.2)$ if spheroids are produced by the major merger of gaseous disks, and if the cold gas is transformed rapidly into stars during the merger in a dynamical time or so. Both conditions should be demonstrated, in particular the latter. A kind of positive feedback seems 
to be necessary for such an efficient star formation rate (ISM shocks produced by the jets generated in the vicinity of supermassive black holes?).

- Once the elliptical has formed early, the next difficulty is how to avoid further (disk) growth around it. The problem can be partially solved by considering that ellipticals form typically in dense, clustered environments, and at some time they become substructures of larger virialized groups or clusters, truncating any possible accretion to the halo/galaxy. However, (i) galaxy halos, even in clusters, are filled with a reservoir of gas, and (ii) there are some ellipticals in the field. Therefore, negative feedback mechanisms are needed to stop gas cooling and accretion. AGN-triggered radio jets have been proposed as a possible mechanism, but further investigation is necessary.

- The merging mechanism of bulge formation within the hierarchical model implies roughly bluer (later formed) disks as the bulge-to-disk ratio is larger, contrary to the observed trend. The secular scenario could solve this problem but it is not still clear whether bars disolve or not in favor of pseudobulges. It is not clear also if the secular scenario could predict the central supermassive black hole mass-velocity dispersion relation.

- We lack a fundamental theory of star formation. So far, simple models, or even just phenomenological recipes, have been used in galaxy formation studies. The two proposed modes of star formation (the quiescent, inefficient, disk self-regulated regime, and the violent efficient star-bursting regime in mergers) are oversimplifications of a much more complex problem with more physical mechanisms (shocks, turbulence, etc.). Closely related to star formation is the problem of feedback. The feedback mechanisms are different in the ISM of disks, in the gaseous medium of merging galaxies with a powerful energy source (the AGN) other than stars, and in the diluted and hot intrahalo medium around galaxies.

- We have seen in $\S \S 2.2$ that at the present epoch only $\approx 9 \%$ of baryons are within virialized structures. Where are the remaining $91 \%$ of the baryons? The fraction of particles in halos measured in $\Lambda \mathrm{CDM} N$-body cosmological simulations is $\sim 50 \%$. This sounds good but still we have to explain, within the $\Lambda \mathrm{CDM}$ scenario, the $\sim 40 \%$ of missing baryons. The question is were these baryons never trapped by collapsed halos or were they trapped but later expelled due to galaxy feedback. Large-scale N-body+hdydrodynamical simulations have shown that the gravitational collapse of filaments may heat the gas and keep a big fraction of baryons outside the collapsed halos 35. Nevertheless, feedback mechanisms, especially at high redshifts, are also predicted to be strong enough as to expel enriched gas back to the Intergalactic Medium. The problem is open.

The field has plenty of open and exciting problems. The $\Lambda$ CDM scenario has survived many observational tests but it still faces the difficulties typical of a theory constructed phenomenologically and heuristically. Even if in the future it is demonstrated that CDM does not exist (which is little probable), the $\Lambda \mathrm{CDM}$ scenario would serve as an excellent "fitting" model to reality, which would strongly help researchers in developing new theories. 
Acknowledgments.- I am in debt with Dr. I. Alcántara-Ayala and R. NúñezLópez for their help in the preparation of the figures. I am also grateful to J. Benda for grammar corrections, and to the Editors for their infinite patience.

\section{References}

1. Abadi, M. G. et al. 2003, ApJ, 591, 499

2. Avila-Reese, V., \& Firmani, C. 2000, RevMexAA, 36, 23

3. Avila-Reese, V., Firmani, C. \& Hernández, X. 1998, ApJ, 505, 37

4. Avila-Reese, V., et al. 2003, ApJ, 598, 36

5. Avila-Reese, V. et al. 2005, ApJ, 634, 51

6. Balogh, M. L. et al. 2004, ApJ, 615, L101

7. Ballesteros-Paredes, J. et al. 2006, in "Protostars and Planets V", in press (astro-ph/0603357)

8. Bartolo, N. et al. 2004, Phys.Rep., 402, 103

9. Baugh, C.M., Cole, S., \& Frenk, C.S. 1996, MNRAS, 283, 136

10. Baugh, C.M. et al. 2005, MNRAS, 356, 1191

11. Bell E. F. et al. 2003, ApJSS, 149, 289

12. Benson, A.J. et al. 2002, ApJ, 599, 38

13. Berlind, A. A. et al. 2005, ApJ, 629, 625

14. Binney, J. \& Tremaine S. 1987, Galactic Dynamics, Princeton Univ. Press, Princeton

15. Blumenthal, G. R. et al. 1984, Nature, 311, 517

16. Boissier, S. \& Prantzos, N. 2001, MNRAS, 325, 321

17. Bond, J. R. et al. 1991, ApJ, 379, 440

18. Bosma, A. 1981, AJ, 86, 1791

19. Bower, R.G. et al. 2005, preprint, astro-ph/0511338

20. Bromm, V., \& Larson, R. B. 2004, ARA\&A, 42, 79

21. Bullock, J. S. et al. 2001, MNRAS, 321, 559

22. Bullock, J.S. et al. 2001, ApJ, 555, 240

23. Carroll S.M., Press W.H., Turner E.L., 1992, ARA\&A, 30, 499

24. Casertano, S., \& van Gorkom, J.H. 1991, AJ, 101, 1231

25. Catinella, B., Giovanelli, R., \& Haynes, M. P. 2005, astro-ph/0512051

26. Cen, R., \& Ostriker, J. 1992, ApJ, 393, 22

27. Ciardi, B. \& Ferrara, A. 2005, Space Science Reviews, 116, 625

28. Cole, S. et al. 1994, MNRAS, 271, 781

29. Cole, S. et al. 2000, MNRAS, 319, 168

30. Coles, P., \& Lucchin, F. 1995, Cosmology. The origin and evolution of cosmic structure, Chichester: Wiley, -c1995,

31. Colín, P., Avila-Reese, V., \& Valenzuela, O. 2000, ApJ, 542, 622

32. Colín, P., Avila-Reese, V., Valenzuela, O., \& Firmani, C. 2002, ApJ, 581, 777

33. Combes, F., preprint, astro-ph/0506265

34. Croton, D. J. et al. 2005, MNRAS, 356, 1155

35. Davé, R. et al. 2001, ApJ, 552, 473

36. Davis, M. et al. 1985, ApJ, 292, 371

37. de Blok, W. J. G. 2005, ApJ, 634, 227

38. De Lucia, G., Kauffmann, G., \& White, S. D. M. 2004b, MNRAS, 349, 1101

39. De Lucia, G. et al. 2006, MNRAS, 366, 499 
40. Dressler, A. 1980, ApJ, 236, 351

41. Eisenstein D. J. et al., 2005, ApJ, 633, 560

42. Eke, V.R., Navarro, J.F. \& Steinmetz, M., 2001, ApJ, 554, 114

43. Fall, S.M. \& Efstathiou, G. (1980), MNRAS, 193, 189

44. Firmani, C., \& Tutukov, A.V. 1994, A\&A, 288, 713

45. Firmani, C., \& Avila-Reese, V. 2000, MNRAS, 315, 457

46. Firmani, C., \& Avila-Reese, V. 20003, RevMexAA (SC), v. 17, 106

47. Flores R.A. et al. 1993, ApJ, 412, 443

48. Fukugita, M., \& Peebles, P. J. E. 2004, ApJ, 616, 643

49. Frenk, C.S. 2002, preprint, astro-ph/0208219

50. Gentile, G. et al. 2004, MNRAS, 351, 903

51. Giavalisco, M. et al. 2004, ApJ, 600, L103

52. Gnedin, O. Y. et al. 2004, ApJ, 616, 16

53. Gondolo, P. 2004, preprint, astro-ph/0403064

54. Goto, T. et al. 2003, MNRAS, 346, 601

55. Gottlöber, S., Klypin, A., \& Kravtsov, A.V. 2001, ApJ, 546, 223

56. Governato, F., et al. 2004, ApJ, 607, 688

57. Gunn, J. E. 1977, ApJ, 218, 592

58. Guzzo, L. 2002, in Modern Cosmology, eds. S. Bonometto, V. Gorini \& U. Moschella, Bristol, UK, 344

59. Hayashi, E., et al. 2004, MNRAS, 355, 794

60. Helly, J. C. et al. 2003, MNRAS, 338, 903

61. Hopkins, A.M. \& Beacom, J. F. 2006, preprint, astro-ph:0601463

62. Hu, W., \& Dodelson, S. 2002, ARA\&A, 40, 171

63. Kang, X. et al. 2005, ApJ, 631, 21

64. Katz, N. 1992, ApJ, 391, 502

65. Katz, N., \& Gunn, J. E. 1991, ApJ, 377, 365

66. Kauffmann, G., White, S.D.M., \& Guiderdoni, B. 1993, MNRAS, 264, 201

67. Kauffmann, G. et al. 1999, MNRAS, 303, 188

68. Kauffmann, G. et al. 2004, MNRAS, 353, 713

69. Kereš, D., Katz, N., Weinberg, D. H., \& Davé, R. 2005, MNRAS, 363, 2

70. Klypin, A.A. et al. 1999, ApJ, 522, 82

71. Kormendy, J., \& Kennicutt, R. C. 2004, ARA\&A, 42, 603

72. Kolb, E.W. \& Turner, M. S. 1990, The Early Universe, Redwood City, California-Addison-Wesley Publishing Company

73. Lacey, C.G., \& Cole, S. 1993, MNRAS, 262, 627

74. de Jong, R. S., \& Lacey, C. 2000, ApJ, 545, 781

75. Lin, Y., Mo, H.J. \& van den Bosch F.C., 2005, astro-ph/0510372

76. Longair, M. S. 1989, LNP Vol. 333: Evolution of Galaxies: Astronomical Observations, 333,1

77. Longair, M. S. 1998, Galaxy formation. Springer-Verlag (Germany)

78. Maller, A.H., Dekel, A., \& Somerville, R. 2002, MNRAS, 329, 423

79. Madau, P., Pozzetti, L., \& Dickinson, M. 1998, ApJ, 498, 106

80. Mandelbaum, R. et al. 2005, preprint, astro-ph/0511164

81. Matos, T., \& Ureña-López, L. 2001, Phys.Rev.D, 63, 063506

82. Mo, H.J., Mao, S., White, S.D.M. 1998, MNRAS, 295,319

83. Moore, B. et al. 1999, ApJ, 524, L19

84. Moore, B. et al. 1998, ApJ, 499, L5

85. Murali, C. et al. 2002, ApJ, 571, 1 
86. Navarro, J. F., \& White, S. D. M. 1993, MNRAS, 265, 271

87. Navarro, J.F., Frenk, C.S., \& White, S.D.M. 1997, ApJ, 490, 493

88. Navarro, J. F., et al. 2004, MNRAS, 349, 1039

89. Nicastro, F., et al. 2005, Nature, 433, 495

90. Padmanabhan, T. 1993, Cosmic structure formation in the universe, Cambridge Univ. Press

91. Patton, D.R. et al. 2002, ApJ, 565, 208

92. Peacock, J. A. 1999, Cosmological Physics, Cambridge University Press

93. Peebles, P.J.E. 1969, ApJ, 155, 393

94. Peebles, P.J.E. 1993, Principles of Physical Cosmology, Princeton: Princeton University Press

95. Persic, M., Salucci, P. \& Stel, F. 1996, MNRAS, 281, 27

96. Postman, M. \& Geller, M. J. 1984, ApJ, 281, 95

97. Postman, M., et al. 2005, ApJ, 623, 721

98. Press, W. H., \& Schechter, P. 1974, ApJ, 187, 425

99. Rubin, V.C., Thonnard, N., \& Ford, W.K. 1980, ApJ, 238, 471

100. Rhee, G. et al. 2004, ApJ, 617, 1059

101. Salucci, P., \& Gentile, G. 2005, preprint, astro-ph/0510716

102. Seljak U. et al., 2005, Phys. Rev. D, 71, 103515

103. Sheth, R. K., Mo, H. J., \& Tormen, G. 2001, MNRAS, 323, 1

104. Silk, J. 1968, ApJ, 151, 459

105. Silk, J. 1977, ApJ, 211, 638

106. Silk, J., \& Rees, M. J. 1998, A\&A, 331, L1

107. Simon, J. et al. 2005, ApJ, 621, 757

108. Somerville, R.S., \& Primack, J.R. 1999, MNRAS, 310, 1087

109. Spergel, D. N. e t al. 2006, prepint, astro-ph/0603449

110. Springel, V., \& Hernquist, L. 2003, MNRAS, 339, 289

111. Springel, V., et al. 2005, Nature, 435, 629

112. Steinmetz, M., \& Müller, E. 1994, A\&A, 281, L97

113. Steinmetz, M. 1996, in Dark Matter in the Universe, Eds. S. Bonometto, J.R. Primack, and A. Provenzale Oxford, GB: IOS Press, 1996, p.479.

114. Tanaka, M., et al. 2004, AJ, 128, 2677

115. Tegmark M. et al., 2004, Phys.Rev. D, 69, 103501

116. Tully, R.B., \& Pierce, M.J. 2000, ApJ, 533, 744

117. Valenzuela, O.\& Klypin, A. 2003, MNRAS, 345, 406

118. Valenzuela, O. et al. 2005, preprint, astro-ph/0509644

119. van den Bosch, F. C. 2000, ApJ, 530, 177

120. van den Bosch, F. C. 2001, MNRAS, 327, 1334

121. van den Bosch, F. C. 2002, MNRAS, 331, 98

122. van den Bosch 2001, F. C., Burkert, A., \& Swaters, R. A. MNRAS, 326, 1205

123. Verheijen, M.A.W. 1997, PhD. Thesis, Groningen University

124. Wang, B., \& Silk, J. 1994, ApJ, 427, 759

125. Wechsler, R.H., et al. 2002, ApJ, 568, 52

126. Weil, M., Eke, V. R., \& Efstathiou, G. P. 1998, MNRAS, 300, 773

127. Weinmann, S. M. et al. 2006, MNRAS, 366, 2

128. Weldrake, D. T. F., de Blok, W. J. G., \& Walter, F. 2003, MNRAS, 340, 12

129. White, S.D.M. 1996, Cosmology and Large Scale Structure, 349, astro-ph/9410043

130. White, S.D.M., \& Frenk, C.S. 1991, ApJ, 379, 52

131. White, S.D.M. \& Rees, M. J. 1978, MNRAS, 183, 341

132. Zavala, J. et al. 2003, A\&A, 412, 633

133. Zhao, D. H. et al. 2003, ApJ, 597, L9 